\title{
Der indische Revolutionär Har Dayal und seine Tonaufnah- men von 1918 im Phonogrammarchiv der Österreichischen Akademie der Wissenschaften ${ }^{1}$
}

Ulla Remmer

\begin{abstract}
In 1918, during his nine-month wait for a visa in Vienna, Har Dayal, the Indian revolutionary, philologist and member of the Berlin-based Indian Independence Comittee, visited the Phonogrammarchiv. The resulting five recordings, containing specimens of literary works recited and sung by Har Dayal in varieties of Hindustani (Hindi, Urdu, Braj Bhasha and Awadhi), were ostensibly made to enlarge the archive's range of languages. In this paper the historical circumstances behind the origins of these recordings as well as their contents will be analysed. It seems that the various literary texts were carefully chosen by Har Dayal himself, as can be judged from their strong biographical affinities. A close scrutiny, moreover, reveals his more or less deliberate attempt at self-portrayal or even self-justification at a pivotal point of his life by means of reciting particular works and mythological tales. In the first part of this study, for the purpose of contextualisation and a better understanding of the recordings, Har Dayal's life will be sketched, largely following Emily C. Brown (1975) and Horst Krüger (1964). In the second part, Har Dayal's months in the AustroHungarian monarchy will be reconstructed, and the network of politics and humanities between Orient and Occident, prevalent in the days around World War I and essential for Har Dayal's activism, will be described. In the third part, the recordings themselves will be investigated.
\end{abstract}

Dieser Aufsatz hätte nicht geschrieben werden können ohne die Hilfe von Suresh und Prerana Chandvankar (Society of Indian Record Collectors, Mumbai), Alaka Chudal (Institut für Südasien-, Tibet- und Buddhismuskunde der Universität Wien) und Stephan Popp (Institut für Iranistik der Österreichischen Akademie der Wissenschaften). Für ihren großzügigen zeitlichen Einsatz - sei es durch genaues Anhören der historischen Aufnahmen und Identifizieren der Texte oder durch sprachliche und historische Expertise und das Verfassen der Übersetzungen in Kapitel 3 - möchte ich mich herzlich bedanken. Navina Sundaram sei für Informationen und Mitteilung eigener Recherchen zu ihrem Großvater Umrao Singh Sher-Gil gedankt. Dank gebührt zudem Meenaxi BarkatakiRuscheweyh (Institut für Ethnologie und Ethnologische Sammlung der Universität Göttingen), Heike Liebau (Zentrum Moderner Orient, Berlin), Ágnes Pap (Országos Széchényi Könyvtár), Antonia Ruppel (Cornell University Department of Classics), Jürgen Schöpf (Phonogrammarchiv der Österreichischen Akademie der Wissenschaften), Chlodwig $\mathrm{H}$. Werba (Institut für Südasien-, Tibet- und Buddhismuskunde der Universität Wien), Annegret Wilke (Politisches Archiv des Auswärtigen Amts, Berlin) und Martin Kümmel (Universität Jena, Lehrstuhl für Indogermanistik). 


\section{Har Dayal und das indologisch-politische Netzwerk während des Ersten Weltkriegs}

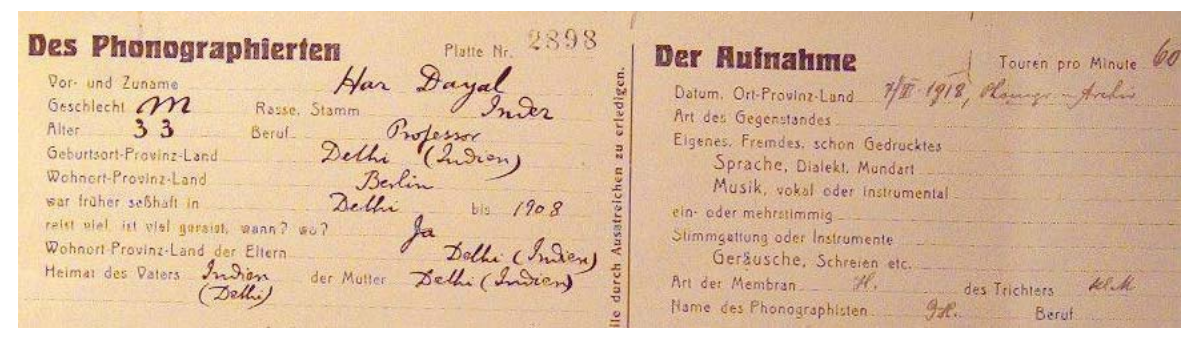

Abb. 1: Kopf des Protokollblatts zu Aufnahme Ph 2898

Ende Jänner 1918 war der indische Unabhängigkeitskämpfer Har Dayal unter dem Decknamen „Professor Mirza Osman“ nach Wien gekommen. Doch im Wiener Phonogrammarchiv, das er nur wenige Tage nach seiner Ankunft besuchte, signierte er mit eigenem Namen. Das obengezeigte Protokollblatt des Phonogrammarchivs, mit standardisierten Fragen zur aufgenommenen Person, war öffentlich kaum zugänglich und damit so unverfänglich, dass sich der Befragte darüber keine Gedanken machte. Auch mag sein wissenschaftlicher Stolz eine Rolle gespielt haben, denn Har Dayal war Experte für die aufgenommenen Sprachen, wenn auch nicht, wie von ihm angegeben, Professor. Bis zu seiner revolutionären Berufung hatte der aus einer gebildeten Hindu-Familie stammende Har Dayal exzellente universitäre Leistungen erbracht, so als Student am St. Stephen's College in Delhi, am Government College in Lahore (Panjab), als Boden-Stipendiat in Oxford, sodann als Lektor für Sanskrit und indische Philosophie in Harvard und in Stanford. Auch später, im schwedischen Exil, wird Har Dayal sich als Professor bezeichnen, obwohl er an keiner Universität beschäftigt war (Brown 1975: 226).

Zu Beginn des letzten Kriegsjahrs suchte Har Dayal das Phonogrammarchiv der kaiserlichen Akademie der Wissenschaften auf, an dessen ursprünglichem Standort im Physiologischen Institut der Universität Wien, Währingerstraße 13. Am 7. Februar 1918 gab Har Dayal, auf fünf Tonaufnahmen verteilt, einige Sprachproben von Hindi und Urdu, rezitierte Lyrik berühmter Dichter, nationalistische Hymnen und aus dem Epos Rāmcaritmānas, erzählte eine Geschichte aus dem Leben des Gottes Krishna und sang einen zeitgenössischen Theaterschlager. Einen Großteil der Kriegszeit zuvor hatte sich Har Dayal im deutschen Kaiserreich aufgehalten und mit den Behörden in Berlin zusammengearbeitet. 
Har Dayal, der einflussreiche Revolutionär, der Indiens Unabhängigkeit erwirken wollte, und der heute wie viele andere Freiheitskämpfer Indiens durch die Gestalt Mohandas Karamchand "Mahatma" Gandhis überstrahlt wird, ist eine schwer fassbare Persönlichkeit. Sein Leben war geprägt von beständigem Lernen, häufigem Wandel in seinen Einstellungen und gleichermaßen häufigem Ortswechsel. ${ }^{2}$ „As a personality“, schreibt seine Biografin Emily Brown (1975: 7),

Har Dayal is elusive. What has intrigued most of those who knew him were the seemingly abrupt changes in his actions and attitudes which occurred as he moved from a militant nationalist - and baiter par excellence of British pretensions - to a pacifist and internationalist who embraced not only the ideals but the homilies of the society he had once scorned and reviled.

Nach Jahren des Sympathisierens wird Har Dayal 24-jährig selbst in der indischen Freiheitsbewegung aktiv. Skandalöserweise legt er Anfang 1908 das ehrenvolle Oxfordstipendium nieder und geht zurück nach Indien, um für einige Monate im Panjab zu agitieren, bis er den britischen Behörden auffällt. Har Dayal, der, wie es in Indien häufig ist, jung geheiratet hat, lässt seine hochschwangere Frau in Indien zurück. Jahre der Propagandaarbeit in Europa wechseln mit längeren Aufenthalten in Marokko, Martinique und Honolulu, wo sich Har Dayal der Meditation widmet. 1913 gründet Har Dayal gemeinsam mit anderen an der Westküste der USA, wo er zuvor an der Stanford University unterrichtet hat und wo viele indische Wirtschaftsmigranten leben, die Ghadr-Partei (von Urdu ghadr "Revolte, Revolution“) mit dem Ziel, Indien von der Kolonialmacht der Briten zu befreien. Har Dayal sympathisiert und experimentiert mit der Gewerkschaftsbewegung, dem Marxismus, Anarchismus, Buddhismus, er pflegt asketisches Fasten und Meditation. In seinem Artikel „Karl Marx: A Modern Rishi“ (Dayal 1912) beschreibt er den wirtschaftlichen Vordenker als einen von Millionen auf der Welt verehrten Heiligen und Weisen.

In den USA schreiten wiederum die amerikanischen Behörden auf Druck der britischen ein. Har Dayal flüchtet im Frühjahr 1914 zunächst in die Schweiz, von wo er nach Ausbruch des Krieges ins deutsche Kaiserreich wechselt, um gemeinsam mit anderen indischen Freiheitskämpfern und mit dem deutschen politischen Establishment von Berlin aus gegen das

Har Dayals Antwort „ja“ auf die Frage nach seiner Reisetätigkeit im Protokollblatt (s. Abb. 1) beinhaltete zum damaligen Zeitpunkt Aufenthalte u.a. im Panjab, in London, Paris, Algier, Martinique, Massachusetts, Kalifornien, Oregon, Honolulu, Genf, Lausanne, Berlin, Konstantinopel, Den Haag, Damaskus und Budapest. 
Britische Empire zu arbeiten. Im Frühjahr 1914 war der Linksaktivist und Unabhängigkeitskämpfer Virendranath Chattopadhyaya nach Deutschland gekommen und hatte mit Abhinashchandra Bhattacharya, der in Halle Chemie studiert hatte, das "Berliner Komitee“, oder Indian Independence Comittee (IIC), gegründet. In enger Zusammenarbeit mit dem Kaiserlichen Auswärtigen Amt in Berlin und den dort für den Orient zuständigen Diplomaten, insbesondere Legationsrat Otto Günther von Wesendonk, werden Pläne für antibritische Propaganda und Aufwiegelungen erdacht und ausgeführt. Neben der Bekämpfung des gemeinsamen Feindes und deutschen Kriegsgegners Großbritannien verfolgt Deutschland seine eigenen Ziele, nämlich nicht nur mit dem verbündeten Osmanischen Reich die englische Vorherrschaft in den Kolonien zu brechen (und dafür die panislamische Bewegung zu stützen), sondern auch in einem eigenen imperialistischen Ansatz deutsche Kolonien im Orient zu gründen. Federführend ist dabei der Diplomat und Orientalist Max von Oppenheim, der Begründer der "Nachrichtenstelle für den Orient", einer quasi-universitären orientalistischen und gleichzeitig nachrichtendienstlich-politischen Institution für propagandistische Tätigkeit, die eng mit dem Auswärtigen Amt zusammenarbeitet (vgl. Krüger 1964: 147ff.). Darin ist für indische Angelegenheiten der Indologe Helmuth von Glasenapp zuständig, der spätere Lehrstuhlinhaber in Königsberg und Tübingen.

Die Allianz aus den indischen Intellektuellen, die vielfach einen linksrevolutionären, anarchistischen Hintergrund haben, und dem wilhelminischen Politik-Establishment ist - so sollte man meinen - eine reine Zweckgemeinschaft (cf. McGetchin 2010: 103). Und doch sind viele Inder, darunter Har Dayal, zunächst auch beeindruckt von dem jungen deutschen Kaiserreich und seiner technischen Vorherrschaft und Effizienz, der deutschen Kultur, Wissenschaft und Philosophie. Darüber hinaus hatte die deutschsprachige Indologie - ganz ohne koloniales Machtgefälle - große Leistungen erbracht, was Sympathien in Indien geweckt hatte (ibid.: 96). Gegenseitig stellte man die Kultur und Philosophie der jeweils anderen Seite als maßgeblich und wichtig für die eigene Geistesgeschichte dar (ibid.: 104f.; Dayal 1918). Schon vier Jahre vor dem Weltkrieg lobt Har Dayal Berlin als "haven of refuge“, wo der Arm des Britischen Empires keinen Einfluss habe: „The cultivation of friendly relations with the powerful German nation will be great advantage to the cause of Indian independence." Tatsächlich hat Deutschland bereits vor dem Ersten Weltkrieg mit Geld und Aktion geholfen, etwa indem deutsche Agenten Ghadr-Schriften in der Welt verbreiteten (Brown 1975: 78, 149-151).

Als 1914 der Krieg ausbricht, ist Har Dayal zunächst nur von seinem Wohnort Genf aus zur Mitarbeit mit den deutschen Behörden bereit. Er reist 
nach Konstantinopel, kehrt aber bald wieder überraschend in die Schweiz zurück. Offenbar gab es Differenzen mit den muslimischen Partnern, aber vor allem fühlte sich Har Dayal von Max von Oppenheim brüskiert (Krüger 1964: 146f.). Chattopadhyaya und Bhattacharya hatten in Berlin unter internierten indischen Studenten weitere Mitglieder rekrutiert, doch im Berliner Auswärtigen Amt wünschte man sich bedeutendere Persönlichkeiten der Nationalismusbewegung und drängte - lange Zeit vergeblich - auf Har Dayal, er möge schnellstens nach Berlin kommen. Vier Monate nach Ausbruch des Ersten Weltkriegs formuliert Har Dayal in einem Brief vom 24. November 1914 an seinen Freund, den Literaturhistoriker und späteren Pulitzer-Preisträger Van Wyck Brooks, zurückhaltende und kritische Gedanken über den Krieg und den in Europa ausgebrochenen "bedrückenden Nationalismus". ${ }^{3}$

Und doch gilt der Satz, ebenfalls in einem Brief wenige Monate zuvor an Van Wyck Brooks formuliert: „I am a revolutionist first and everything else afterward." (Brown 1975: 108). Har Dayal lässt sich vom I/C überzeugen und trifft am 27. Jänner 1915 in Berlin ein, worauf er unverzüglich mit Propagandaaktivitäten und Vorbereitungen zur Revolte beginnt. 1915 verbringt Har Dayal wiederum einige Monate in Konstantinopel, diesmal um die Mission des Raja Mahendra Pratap (1886-1979) vorzubereiten. Der indische Aristokrat, der aus Berlin im Frühjahr nach einem Treffen mit dem Ex-Khediven von Ägypten in Wien nach Konstantinopel gefahren war, sollte mit Unterstützung der Deutschen vom Bosporus aus durch englisch kontrolliertes Gebiet nach Kabul reisen, um den afghanischen König zur Zusammenarbeit zu bewegen (Brown 1975: 189). Die Mission (die sogenannte NiedermayerHentig-Expedition) erreichte zwar ihr Ziel, doch der verhandlerische Erfolg in Kabul war bescheiden (s.u.). Ebenfalls erfolglos blieben die Aufstandsversuche im Frühjahr 1916 im nordindischen Panjab und in Bengalen, für die viele

Vgl. Brown (1975: 186f.):

\footnotetext{
I don't know, but I think that the next half century will be marked by great reaction all around. This war has been the grave of many reputations and movements. It is sad to see how the learned and wise men mingle in the strife. Learning should be above all such partisan blindness. I think Haeckel and all of them, among all nations, have cut a rather sorry figure. In USA, they are taking sides for the Triple Entente. I say, "a plague o' both of your houses!" It is a war of rival commercial groups. What have I or the poor people got to do with it? [...] Here on the continent sane thought is nonexistent. [...] People are for or against Germany. That is all. [...] No one has taken up any sociological standpoint, discussing the issues involved. It is rather disappointing to see that there is now no philosophical class in Europe, which should be able to take large views and interpret human interests. I feel cramped and choked in this atmosphere. Even Kropotkin has gone the way of the rest. I am afraid that the invasion of all the nooks and corners of intellectual life by patriotism bodes ill for the future of European thought. The race of Erasmus, Goethe, Marx, should not become extinct. The theory of national "cultures" is being heard of a great deal.
} 
indische Arbeiter sowie Mitglieder und Sympathisanten der Ghadr-Partei, von Har Dayal dazu bewogen, aus den USA nach Indien zurückkehrten.

Auch im Jahr 1915 können Har Dayal und die muslimischen Verhandlungspartner in Konstantinopel keine gemeinsame Arbeit aufbauen. Den muslimischen Indern fällt der ausgeprägte Führungsanspruch des Hindus negativ auf, und über die türkischen Partner verliert er selbst böse Worte. Die deutsche Seite kann nicht vermitteln, denn Har Dayal fühlt sich von den Diplomaten seinerseits schlecht behandelt und kritisiert. Zunehmend sieht Har Dayal auch imperialistische und rassistische Attitüden in seinen deutschen Verbündeten und Brotgebern, die eine Kolonialisierung Kleinasiens durch deutsche „Herrensiedler" anstreben (Krüger 1964: 147; McGetchin 2010: 96).

Ohne Ankündigung und zur Erleichterung der Diplomaten verlässt Har Dayal Ende August 1915 Konstantinopel und reist allein nach Budapest, um Umrao Singh Sher-Gil Majithia (1870-1954), einen weiteren mit der Unabhängigkeitsbewegung sympathisierenden indischen Aristokraten, zu treffen. Dieser, ein Sirdar (Sardar) aus Majitha, hatte vor dem Krieg in Lahore gelebt, wo inm Har Dayal vermutlich schon während seiner Studienjahre begegnet war. Wie Har Dayal ist der Sirdar ein guter Philologe, u.a. für Sanskrit, Urdu und Persisch. Seit Kriegsausbruch lebt Umrao Singh Sher-Gil, dessen Güter in Indien konfisziert wurden, mit seiner ungarischen Ehefrau und den beiden Töchtern in Budapest und Umgebung. Heute ist er vor allem als Vater der Malerin Amrita Sher-Gil (1913-1941) und als Freund des indischmuslimischen Dichters Muhammad Iqbal bekannt (dazu und zu den Tonaufnahmen, die von Umrao Singh Sher-Gil im Wiener Phonogrammarchiv erhalten sind, s.u.).

Har Dayal versucht mit erheblichem Aufwand, Umrao Singh Sher-Gil zur aktiven Mitarbeit im Berliner Komitee (IIC), etwa zur Beeinflussung der Sikhs in den Kriegsgefangenenlagern, zu bewegen und lädt ihn nach Berlin ein: Zwischen 4. und 18. Oktober wird der Sirdar von hochrangigen Beamten und Diplomaten empfangen und trifft namhafte Indologen aus Berlin und Umgebung, wie Glasenapp, Heinrich und Else Lüders und - laut Programm - Paul Deussen. ${ }^{4}$ Ein Besuch der indischen Kriegsgefangenen im „Halbmondlager" bei Wünsdorf war geplant und vermutlich auch ausgeführt worden. Doch die höflichen Dankesworte des Sirdars an den Diplomaten von Wesendonk

Der einflussreiche Philosoph und Indologe Paul Deussen aus Kiel hatte viel zur Bekanntwerdung der indischen Philosophie im Westen beigetragen und dem erwachenden Neo-Hinduismus in Indien Impulse gegeben. 
vom 17. Oktober ${ }^{5}$ beinhalten eine Absage für Berlin, gewiss auch von der Ehefrau Umrao Singh Sher-Gils, Marie Antoinette geb. Gottesmann, beeinflusst, der das politische Engagement ihres Mannes Sorge um das Wohl der Familie bereitet (AA Wk 11f. R 21089-FN4 BI. 310).

Har Dayals Pläne und Alleingänge stoßen bei Chattopadhyaya und den Kollegen im Komitee auf Kritik. Nach selbständig geplanten Aufenthalten in Den Haag und Damaskus bleibt er ab Anfang 1916 der Gruppe fern und hält sich bis Sommer 1917 unter verschiedenen Namen an wechselnden Adressen u.a. in Wiesbaden, Garmisch-Partenkirchen und im Taunus auf, um in Kurbädern seine Neurasthenie zu kurieren. Das Auswärtige Amt, so schreibt er 1920 in seiner großen Abrechnung mit Deutschland (Dayal 1920), habe inn nicht ausreisen lassen, er sei ein Gefangener im Land gewesen. In der Tat hatte Har Dayal Anfang 1916 geplant, in die Schweiz zu reisen, das Auswärtige Amt jedoch ließ ihm seine drei Pässe abnehmen und wies die Grenzbehörden unter Nennung verschiedener bislang verwendeter Decknamen Har Dayals an, ihn unter keinen Umständen in die Schweiz ausreisen zu lassen, und dies, obwohl die Rückkehr nach Genf ursprünglich zugesichert worden war (AA Wk 11f. R 21074-1 BI. 81). Im April 1916 sucht Har Dayal wieder um einen Pass an, um in die Schweiz zu reisen, man teilt ihm mit, dies sei unmöglich, da er dort sofort ausgewiesen würde. ${ }^{6}$

Im April 1917 äußert Har Dayal den Wunsch, sich wieder zu engagieren. Trotz der früheren Meinungsverschiedenheiten wird im Herbst beschlossen, ihn für propagandistische Arbeit und zur Aussprache zu Chattopadhyaya zu entsenden, der sich gerade in Stockholm aufhält. Als Verschleierungsmaßnahme soll Har Dayal ab Ende Jänner 1918 in Wien auf die Einreisegenehmigung des neutralen Schwedens warten (Krüger 1964: 160). Doch erst im Oktober, einen Monat vor dem Waffenstillstand der Alliierten und Deutschlands, darf Har Dayal in Schweden einreisen, wo er ohne Verzug seine politische Neuausrichtung vorbereitet: In einem am 4. Dezember publizierten Artikel kritisiert er in einer US-amerikanischen Zeitung scharf die in Deutschland herrschende „Junkerklasse“ für ihre von Imperialismus durchdrungene Politik und gratuliert Amerika, diese

Mikrofilmsammlung des Politischen Archivs des Deutsches Auswärtigen Amts, Abt. IA, Aktenreihe Weltkrieg 11 f. (im Folgenden abgekürzt: AA Wk 11f.) Bd. R 21090-FN2 BI. 157.

$6 \quad$ Die Grenzsperre sollte später in Wien für Verwirrung sorgen. In einer handschriftlichen Note als Antwort auf eine Anfrage der amtlichen Stelle „Passzentrale“ zur Ausformulierung und Reinschrift schreibt Legationsrat von Wesendonk um den 14. April 1918: „[...] dass die Ausreise des Mirza Osman (Har Dayal) nach Österreich-Ungarn im vorliegenden Fall im Einverständnis mit dem Ausw. [-ärtigen] A[mt] erfolgte und daher unbedenklich ist. Har Dayal darf nur nicht die Möglichkeit haben, nach eigenem Gutdünken Deutschland zu verlassen. Nur aus diesem Grunde war die Grenzsperre für ihn seinerzeit[?] beantragt worden." (AA Wk 11f. R 21113-2 Bd. 43 BI. 199f.). 
arrogante Nation erniedrigt zu haben (Brown 1975: 218). 1920 wird Har Dayal noch ausführlicher und publiziert den Essay Forty-four months in Germany and Turkey (Dayal 1920), worin er sich den moderaten Strömungen der Home Rule Bewegung anschließt. Er befürwortet nun britische Kolonialherrschaft im Orient, sieht Indien noch nicht reif für Unabhängigkeit, plädiert daher für eine gemischte Verwaltung, und preist die „moral and historical mission“ Englands in Asien. Das Britische Empire sei das kleinere Übel in dem gegenwärtigen Spiel um Einfluss und Kolonien in Asien und Afrika (Dayal 1920: 97-101). Seine Äußerungen werden von den Briten tausendfach gedruckt und in Indien verbreitet. ${ }^{8}$

Zweifellos war Har Dayal in einer seelischen Grenzsituation. Nach Jahren der "falsehood and dissimulation in self-defence“ (Dayal 1920: 74), abhängig von einem Land, das er verachtete, wird sich der Aktivist befreit gefühlt haben, als er endlich neutralen schwedischen Boden betreten hatte. Er, von dem es legendenhaft hieß, er sei 1908 wie ein wandernder Mönch der Freiheit durch den Panjab gewandert, um die Menschen aufzurütteln (vgl. Brown 1975: 51), der Philosoph und Asket, der vor dem Krieg in Martinique in einer Berghütte und in Honolulu in einer Höhle am Strand von Waikiki meditiert hatte, war einer von ihm als erstickend empfundenen

Auch privat drückt er seine Verachtung für die Mittelmächte aus: „I was practically interned in Germany during the last three years and could not correspond with my friends", heißt es zwei Wochen nach seiner Ausreise aus Österreich in einem Brief an seinen Freund Van Wyck Brooks (Brown 1975: 219):

I managed to get out of that absurd country last week, but I still find myself in a difficult situation here. I have suffered and learned and thought much since I wrote to you from Switzerland in 1914, and I may say that sorrow and wisdom have grown in me like twin-sisters. Of course, the world-tragedy of the war must play a part in every man's moral and intellectual development. [...] German ambition, greediness and belief in Force constitute a serious menace to Europe and Asia. Germany must be taught that her dreams of "World-Power" cannot be realized, so that she may direct her colossal energies into more fruitful channels. It is a vigorous and capable people but the Junker class has infected the whole country with its Pan-Germanic mania. [...] Prussian and Austrian imperialism must be abolished, along with the medieval dynasties of the Hohenzollerns and the Hapsburgs, if possible.

\& Forty-four months in Germany and Turkey wird für seinen allzu persönlich gefärbten, beleidigten Grundton zurecht kritisiert; es beeinhaltet unbelegte Vorwürfe gegenüber namentlich genannten und ungenannten Einzelpersonen und dem deutschen Establishment, gegenüber den türkischen Partnern, aber auch den indischen Landsleuten, deren Arbeit in Berlin er abkanzelt. In seinen Darstellungen der Mittelmächte spiegelt Har Dayal die antideutsche Propaganda der alliierten Westmächte der vergangenen Kriegsjahre. Auf der anderen Seite lesen sich einige Passagen heute und im Rückblick auf den Zweiten Weltkrieg geradezu prophetisch: „They are cruel and brutal towards the conquered, as we are cruel to animals, which we slaughter without remorse or compunction for our advantage." Wegen seiner Überheblichkeit und Grausamkeit dürfe Deutschland unter keinen Umständen erlaubt werden, Kolonien zu haben, es würde mit seiner technischen Überlegenheit die Menschen rassistisch und voll Habgier behandeln (Dayal 1920: 15-19). 
bürokratischen Atmosphäre entkommen. Seine Nerven, so sahen es Zeitgenossen und so äußerte er sich auch selbst, waren angeschlagen. Gewiss verband er seine Worte mit der Hoffnung, England würde ihm Amnestie erweisen. Seine Sorge um seine Zukunft ist auch dokumentiert in einem Brief an von Wesendonk, in dem er darum ersucht hatte, nach dem Krieg ein Bleiberecht in Deutschland erhalten zu können (Krüger 1964: 168). Auch wird vermutet, dass Har Dayal die Tatsache zu schaffen machte, dass er niemals wie so viele seiner Weggefährten, die von den Briten zum Tode verurteilt oder auf die Andamanen deportiert waren, direkt durch die Kolonialmacht hatte leiden müssen, denn jeder drohenden Verhaftung hatte er sich durch Ortswechsel entzogen (Brown 1975: 226).

Sehr ärmlich lebt der Exilant in den folgenden Jahre in Schweden von prekären Lehraufträgen über Indien, er lernt Griechisch und Latein, überlegt eine Biografie von Jesus zu schreiben und eine philosophische Akademie in Athen zu gründen, publiziert über das Schul- und Universitätswesen in Indien, über Yoga und die Zukunft des Hinduismus, geißelt öffentlich das Kastenwesen, das er für ein Grundübel Indiens hält (ibid.: 235), aber auch die Kinderheirat, die Abschottung von Frauen (purdah), Polygamie und Analphabetismus.

Har Dayal kann nun Kontakt zu seiner Frau und seiner Tochter, die er nie gesehen hat, aufnehmen. Fortan wird er immer wieder um Amnestie und die Möglichkeit, nach Indien einzureisen, ansuchen, doch lediglich nach England darf er zehn Jahre später mit seiner schwedischen Gefährtin Agda Erikson ziehen. In London macht er sein Doktorat mit der Dissertation The Bodhisattva Doctrine in Buddhist Sanskrit Literature (Dayal 1932), bis heute ein Standardwerk. Er gründet ein philanthropisch-philosophisches Lehrinstitut zur richtigen Lebensführung auf der Basis seines zweiten Buches Hints for Self-Culture (Dayal 1934). Statt politischer und revolutionärer macht er nun philosophische und ethische Propaganda mit pazifistischer und kosmopolitischer Ausrichtung (Brown 1975: 244ff.). ${ }^{9}$

In mancher Hinsicht hatte sich seine Weltanschauung schon immer mit der Gandhis gedeckt, wie etwa in der bereits 1908 von Har Dayal formulierten Mahnung, die Inder trügen selbst Verantwortung für ihre schlechte Lage, und dass die Engländer im Land seien, habe sich Indien auch selbst zuzuschreiben. Wie Gandhi verurteilte Har Dayal das Kastenwesen und propagierte die Nicht-Kooperation (Brown 1975: 69f., 92). Jedoch entwickelte

Har Dayals innere Kehrtwendung wurde nicht nur von den britischen und britisch-indischen Behörden angezweifelt: Auch in seinem persönlichen Umfeld blieb bei manchen der Eindruck, Har Dayal habe seine anti-englische Haltung nie abgelegt (vgl. Brown 1975: 242, 262-265; Paul \& Paul 2003: 147-150). 
Gandhi das Konzept der Gewaltlosigkeit satyagraha (ibid.: 54, 71). ${ }^{10}$ Und während Gandhi die Versöhnung zwischen Hindus und Moslems in einem gemeinsamen Indien als unabdingbar und möglich ansah, war Har Dayals Verhältnis zum muslimischen Teil seines Landes zwiespältig. Der Urdu-Student fiel in jungen Jahren durch seinen ausgeprägten Hinduismus auf (ibid.: 49). Aber zunächst tritt die Frage nach dem Verhältnis von Hindus und Moslems durch die Konzentration auf einen gemeinsamen Feind in Gestalt der Kolonialmacht in den Hintergrund. Ausgerechnet er, der das Oxford-Stipendium der britischen Krone als inakzeptabel niedergelegt hatte, formulierte optimistisch in einem Artikel, der Konflikt zwischen Moslems und Hindus sollte durch die gemeinsamen Werte der im Westen ausgebildeten Anführer beider Seiten lösbar sein (,[The] same force that takes students of both communities to Oxford and Harvard will make them friends at Hyderabad", vgl. Brown 1975: 118). In seiner Tätigkeit während des Ersten Weltkriegs machte sich Har Dayal jedoch bei den muslimischen Partnern äußerst unbeliebt (s.o.). Und in einem 1925 erschienenen Artikel schilderte er in scharfen Worten die Einheit der Hindu und Moslem-Religion als für die Zukunft Indiens unrealistisch und stellte Gandhis Versöhnungsweg völlig in Abrede, indem er u.a. kategorisch erklärte, dass dreizehn Jahrhunderte islamischer Geschichte und tausend Jahre Krieg zwischen Afghanistan und Indien nicht durch ein 21-tägiges Fasten des Mahatmas weggefegt werden könnten (ibid.: 234).

Gegen Ende seines Lebens jedoch suchte Har Dayal hauptsächlich nach Mitteln zu einer Verständigung zwischen Ost und West und skizzierte in seinen Schriften eine Welt, in der sich Kolonialismus und Imperialismus, aber auch Nationalismus auflösen und Frieden und Verständigung weichen würden (Brown 1975: 11). 1938 reiste Har Dayal zu einer längeren Vortragsreise in die USA, während der er am 4. März 1939 in Philadelphia starb, kurz nachdem ihn die Nachricht erreicht hatte, die britisch-indischen Behörden hätten seine Einreise nach Indien gestattet.

\section{Har Dayal in Österreich-Ungarn}

Die Frage, wann Har Dayal seinen politischen Gesinnungswandel innerlich vollzogen hat, wurde von Krüger (1964: 165-167) diskutiert, der vor allem Har Dayals Aufsatz "Der Orient und die deutsche Kultur", publiziert Mitte Juni 1918 in der Zeitschrift Der neue Orient (Dayal 1918), betrachtete.

10 Von manchen wird Har Dayal heute unter die Vordenker des modernen Indiens eingereiht. Zumindest war seine Vorstellung des zukünftigen Staates Indien dem heutigen Zustand näher als das, was Gandhi im Sinn hatte. Anders als Gandhi war Har Dayal für die Entwicklung westlicher Techniken und Institutionen und das Vorantreiben der Industrialisierung (Brown 1975: 3, 6-8, 125). 
Obwohl es sich dabei um eine halb-offizielle Publikation der Nachrichtenstelle für den Orient unter der Ägide des Berliner Auswärtigen Amts handelte, geht Har Dayal, wenn auch nur in manchen Sätzen, zum kriegführenden Deutschland auf vorsichtige Distanz. Bei seiner Erörterung der Faktoren, die für die Macht und Stärke Deutschlands maßgeblich seien, schiebt Har Dayal die militärische und technische Dominanz beiseite und kommt auf das Sozialwesen, die Wissenschaft und vor allem die Kultur. ${ }^{11}$

Hingegen schimmert Reservation durch bei Begriffen, die die deutsche Kriegsgegenwart abbilden (ibid.: 261, 264):

Hat Deutschland dem Orient wirklich etwas zu sagen, oder beschränken sich unsere Beziehungen nur auf diplomatische, militärische und wirtschaftliche Gebiete? Hört wirklich die Verbindung mit Deutschland auf, wenn weitberühmte Namen wie "Generalstab", "Wilhelmstraße" [die Adresse von Regierungsämtern in Berlin, darunter das Auswärtige Amt], "Krupp" und "A.E.G." ihre Bedeutung für uns verloren haben? Oder sollten wir nicht weiter und tiefer gehen und nachforschen, was die Namen, wie Weimar, Königsberg, Heidelberg und Bayreuth für uns bedeuten? Diese Namen bergen vielleicht Schätze von nie endendem Werte für den hungrigen und durstigen Orientalen. [...] Asien sagt zu den Deutschen: „Gebt uns das Beste, was euer Land produziert!" Und es ist unbestreitbar, daß Goethes und Schillers Werke ein besseres Produkt deutscher Arbeit sind als Farbstoffe und Maschinen.

Als diese Zeilen in Druck gingen, war Har Dayal bereits vier Monate in Österreich-Ungarn. Wenn es auch wahrscheinlich ist, dass der Aufsatz

\footnotetext{
1 Vgl. Har Dayal (1918: 262; s. Anm. 19):
}

In gewissen Fällen ist Macht vorübergehend. Es ist möglich, daß ein Volk im Lauf der Zeit bestimmte Elemente seiner Kultur verliert, die zu seinem politischen Wachstum und seinem militärischen Erfolg beigetragen haben. Aber die ganze Kultur einer großen Nation kann niemals verloren gehen. Ihre Literatur, ihre Religion und Philosophie, ihre Kunst, die Lebensbeschreibungen ihrer großen Männer und Frauen - all das ist von ewigem Wert für die Menschheit. Mag selbst der Name einer Nation verschwinden, ihre Kultur wird leben und unter noch ungeborenen dankbaren Generationen blühen. Auf viele Dinge "Made in Germany" sollen wir Orientalen unsere Aufmerksamkeit lenken. Es ist natürlich wichtig zu lernen, wie Waffen und Munition, Messer und Gabeln und chemische Farbstoffe hergestellt werden. Aber es ist viel wichtiger, die deutsche Kultur zu verstehen und den Fortschritt der Zivilisation schätzen zu lernen [...].

Hervorgehoben werden unter den Philosophen Kant, Fichte, Hegel, Feuerbach, Schopenhauer und Nietzsche. Aus der deutschen Literatur nennt Har Dayal neben seinen Idolen Goethe und Schiller die Vertreter der „Poesie der Freiheit“ Ernst Moritz Arndt, Körner, Schenckendorf, Herwegh, Freiligrath sowie die Dramatiker Lessing, Grillparzer und Hebbel, deren Meisterwerke „auf den Bühnen Asiens noch in Jahrhunderten gespielt werden werden." In der deutschen Musik werden Beethoven und Richard Wagner hervorgehoben. 
während seiner erzwungenen Wartezeit an der Donau verfasst wurde, so wird die - hier verhalten und später stark formulierte - kritische Einstellung vermutlich nicht erst in Wien entstanden sein. Ob und inwieweit das Dreivierteljahr in Österreich-Ungarn unmittelbar vor seiner Kehrtwendung Har Dayal geprägt hat, lässt sich nicht sagen. Nur wenige Zeitdokumente sind erhalten, und diese waren hauptsächlich über das Berliner Auswärtige Amt gegangen, sodass ihr Inhalt einseitig ist. Insgesamt lässt sich der Aufenthalt in Österreich nur lückenhaft rekonstruieren.

Schon die Ausreise aus Deutschland in das verbündete ÖsterreichUngarn gestaltete sich offenbar umständlich, doch kurze Zeit nach dem 19. Jänner dürfte Har Dayal alias Professor Mirza Osman in Wien eingetroffen sein (AA Wk 11f. R 21111-1 BI. 68-73). Über die Kaiserlich Deutsche Botschaft in Wien III., Metternichgasse, ist Har Dayal mit dem Auswärtigen Amt verbunden; seine Korrespondenz läuft - zumindest teilweise - über die Botschaft. Auch in der diplomatischen Post wird zumeist der Deckname verwendet, und sein Aufenthalt wird als "ganz geheim" behandelt (cf. u.a. AA Wk Nr. 11f. R 21111-1 BI. 68-70 Note von Wesendonks; R 21111-2 BI. 109 Telegramm des deutschen Botschafters Wedel an Wesendonk). ${ }^{12}$

Kaum angekommen, werden Maßnahmen ergriffen, ihm „als Perser, der in seine Heimat zurückreisen möchte" (AA Wk 11f. R 21111-1 BI. 72) eine Einreisegenehmigung ins neutrale Schweden zu erwirken. Zunächst soll ein persischer Pass die Ausreise ermöglichen (cf. u.a. AA Wk 11f. R 21111-2 BI. 125). Zwar hatte Har Dayal in den Jahren zuvor bereits drei persische Pässe besessen, doch die Erneuerung bzw. Neuausstellung kommt in Wien beim persischen Konsulat nicht voran (AA Wk 11f. R 21112-2, darin u.a. BI. 98, 111, 183). Seine Zweifel an der Ernsthaftigkeit, mit der das Auswärtige Amt seine Ausreise nach Schweden betreibt, drückt Har Dayal in einem Brief an Bhupendranath Datta (1880-1961), damals Sekretär des IIC, vom 29. März aus (AA Wk 11f. R 21113-1 BI. 25f.), worin er beklagt, dass Berlin sich weigere, offizielle Schritte in Teheran in Sachen Pass zu tätigen. Wenig später lässt Har Dayal über das IIC in einem Brief vom 11. April das Auswärtige Amt bitten, „ihn im Pass als deutschen Untertan oder deutschen Schutzgenossen oder wenn dies nicht angängig ist als Afghanen zu bezeichnen“; von diesen

Auch heute ist Har Dayal alias Mirza Osman in keinem Wiener Melderegister aus dem Jahr 1918 nachweisbar (nach Auskunft von Erich Denk, Wiener Stadt- und Landesarchiv, 9.4.2014). Auf seinen Ausreisepapieren vom September steht als „gegenwärtiger Aufenthaltsort“ Wien I. Hotel de France vermerkt, jedoch sind in dem bis heute existierenden Wiener Hotel keine Aufzeichnungen aus dieser Zeit erhalten (Auskunft von Michael Pohn, General Manager Hotel de France, 1010 Wien; 24.3.2014). Es ist zu bedenken, dass sich während des Ersten Weltkriegs viele Flüchtlinge in Wien sammelten, die Wiener Hotels überbelegt waren und Hotelmeldungen aus dieser Zeit nicht vollständig erhalten sind. 
Möglichkeiten scheint, wie aus der handschriftlichen Notiz von Wesendonks hervorgeht, nur die letzte durch das Auswärtige Amt erwogen worden zu sein (AA Wk 11f. R 21113-1 Bl. 159). Später wird überlegt, ihn illegal oder mit einer russischen oder türkischen Identität nach Schweden zu bringen.

Har Dayal steckt nun für einige Monate in Österreich fest. Von seinen Aktivitäten ist wenig bekannt. Es ist anzunehmen, dass er so wie in allen Stationen seines Lebens Bibliotheken aufsucht, viel liest und diskutiert. Eine der wenigen nachweisbaren Aktivitäten während seines Moratoriums in Wien ist der Besuch des Phonogrammarchivs, bereits am 7. Februar, wie aus dem Protokollblatt von Phonogramm 2898 hervorgeht (s. Abb. 1). Har Dayal hat seine biografischen Daten selbst handschriftlich ausgefüllt, der „Phonographist“ des Instituts übernahm den Rest und hinterließ ein Kürzel für den eigenen Namen, vermutlich Dr. H., für den Physiker Dr. Leo Hajek, den damaligen Assistenten und späteren Leiter des Archivs. „Hajek“ ist auch mit Bleistift von anderer Hand am oberen Blattrand vermerkt. Der Gründer und damalige Obmann des Phonogrammarchivs, Sigmund Exner, schrieb noch im selben Jahr in den Tätigkeitsbericht des Archivs im Almanach für das Jahr 1918 der Akademie der Wissenschaften (Exner 1918: 302): „Auf fünf Platten wurden mehrere Aufnahmen hindostanischer Sprachproben in der eigentümlichen Rezitationsweise dieses Volkes festgehalten. Sprecher war Professor Har Dayal aus Delhi.“

Es ist nicht bekannt, wie der Kontakt Har Dayals zum Phonogrammarchiv zustande gekommen ist; angesichts der damaligen Vernetzung von Wissenschaft und Politik ist der Besuch aber keinesfalls verwunderlich. Die Institution hatte sich vor dem Weltkrieg in indologischen Kreisen durch ihre Sammlung von Tonaufnahmen mit brahmanischer Rezitation, aufgenommen vom Meteorologen Felix Exner in den Jahren 1904 und 1905, einen Namen gemacht. Kurz vor dem Krieg war eine ausführlichere Publikation dazu erschienen (Felber \& Geiger 1912). Der Philologe Har Dayal, der zehn Jahre zuvor Indien verlassen hatte, mag sich schon seit längerem für die Aufnahmen interessiert und die Gelegenheit zu einem Besuch im Phonogrammarchiv genutzt haben. Sein Freund und Weggefährte, der bereits erwähnte, in Budapest lebende Sirdar Umrao Singh, von dem das Phonogrammarchiv ebenfalls einige Aufnahmen hat (s.u., Kapitel 3), war persönlich bekannt mit Henry Steel Olcott, dem Präsidenten der Theosophischen Gesellschaft und Begründer der Adyar Library in Madras (heute Adyar Library and Research Centre in Chennai), jener seit ihrer Gründung 1886 für die Indologie wie 
für die Antikolonialismusgeschichte Indiens so bedeutsamen Institution. ${ }^{13}$ Felix Exner hatte den Großteil seiner Phonogramme von 1905 in der Adyar Library aufgenommen und dabei auch Olcotts Stimme mit einer Grußadresse an den Präsidenten der Österreichischen Akademie der Wissenschaften verewigt (Ph 452). Umrao Singh Sher-Gil, der als technikbegeisterter Mensch bekannt war, könnte von Olcott (der allerdings schon 1907 starb) vom Phonogrammarchiv gehört haben. ${ }^{14}$ Selbstverständlich profitierte das Archiv von den Besuchen Har Dayals und Umrao Singh Sher-Gils, denn von den 68 Exner-Phonogrammen beinhalteten die allermeisten Rezitationen von Sanskrit-Werken, hingegen gab es nur wenige Aufnahmen mit neuindischen Sprachen, darunter eine einzige auf Hindi. Für die Archivleitung war es Gelegenheit, die Sammlung zu erweitern.

Zusätzlich ist zu beachten, dass die Hauptaktivität des Phonogrammarchivs während des Ersten Weltkriegs darin bestand, in Kriegsgefangenenlagern Tonaufnahmen von den dort Internierten zu erstellen (vgl. Lange 2013: 321-447), wodurch es zu einem Kontakt mit der indischen Gruppe in Berlin gekommen sein mag. Zwar wirkten die planenden und ausführenden Wissenschaftler (darunter v. a. der Ethnologe und Anthropologe Rudolf Pöch, der Musikwissenschaftler Robert Lach sowie die Mitarbeiter des Archivs Leo Hajek und Hans Pollak) vor allem in den Lagern der österreichisch-ungarischen Doppelmonarchie, jedoch bestand selbstverständlich ein Austausch mit den deutschen Kollegen des Berliner Phonogrammarchivs, die ihrerseits große ethnologische Sammlungen in den Kriegsgefangenenlagern des deutschen Kaiserreichs anlegten. Rudolf Pöch, dessen Arbeit sich nicht auf Tonaufnahmen beschränkte, sondern auch anthropologische Körper-Vermessungen, inklusive Gipsabdrücke der Köpfe, Fotografien und Filmaufnahmen einschloss, war zumindest von August bis Oktober 1917 auf Einladung der deutschen Kollegenschaft für einige Zeit im bekannten "Halbmondlager" bei Wünsdorf tätig (dort jedoch ohne Tonaufnahmen zu machen, vgl. Lange 2013: 108-112). In diesem Lager, das bereits in Zusammenhang mit dem von Har Dayal organisierten Besuch Umrao Singh Sher-Gils in Berlin erwähnt wurde und das ein Hauptzentrum für Propagandaaktivitäten des $/ / C$ und der

131898 schrieb Olcott in seinem Tagebuch Old Diary Leaves von einem Abendessen bei dem Sirdar in Lahore; vgl. <http://www.theosophy.ph/onlinebooks/odl/odl619.html> und <http://www. theosophy.ph/onlinebooks/odl/odl620.html> (30.3.2014).

14 Im Jahr 1918 jedoch, elf Jahre nach Olcotts Tod, lag die Leitung der Theosophischen Gesellschaft und Adyar Library in Madras in den Händen der Engländerin Annie Besant. Für sie, die sich in der indischen Unabhängigkeitsbewegung an höchster Stelle engagiert hatte, äußerten Umrao Singh Sher-Gil und Har Dayal Misstrauen und Verachtung (AA Wk 11f. R 21112-FN1 BI. 55-66, Brief Umrao Singhs an von Wesendonk vom 17.2.1918, darin 56-58; Brown 1975: 70). 
Nachrichtenstelle für den Orient bildete, waren zahlreiche indische Kriegsgefangene interniert, und in ihm führten u.a. auch der bereits erwähnte Berliner Indologe Heinrich Lüders und der Indogermanist Wilhelm Schulze ethnografische und sprachwissenschaftliche Untersuchungen durch. Es ist gut denkbar, dass Har Dayal, dem die Kriegsgefangenen aus Indien ein Anliegen waren, den Österreicher Pöch, der vor dem Krieg Assistent im Wiener Phonogrammarchiv gewesen war, und andere Ethnologen und Phonographisten in Wünsdorf getroffen oder zumindest von ihnen gehört hatte. ${ }^{15}$

Den Besuch Har Dayals im Phonogrammarchiv könnte nicht zuletzt auch das Institut für Orientalistik der Universität Wien und dessen Ordinarius für Indologie, Leopold von Schroeder, vermittelt haben. Der aus dem Baltikum stammende Orientalist, Mythenforscher und Dichter von Schroeder, der maßgebliche Editionen altindischer Texte und ein umfangreiches wissenschaftliches Werk geschaffen hatte, kannte selbstverständlich die Indologen in Berlin und Umgebung. Zudem hatte von Schroeders Mitarbeiter Bernhard Geiger, seit 1909 „Privatdozent für altindische und altiranische Philologie und Altertumskunde", kurz zuvor im Wintersemester 1917/18 erstmalig in seiner Laufbahn Hindustani unterrichtet, und er plante einen Lektüre-Kurs im folgenden Sommersemester (cf. Bihl 2009: 85). Har Dayals Expertise muss Geiger willkommen gewesen sein. Geiger war zudem mit dem Phonogrammarchiv bestens vertraut, da er es gewesen war, der die Phonogramme der Exner-Sammlung hinsichtlich ihrer Texte bearbeitet hatte (Felber \& Geiger 1912). Es ist nicht belegt, ob Geiger bei den

5 Har Dayal, der nach dem Krieg den Rassismus in Deutschland anprangerte (vgl. oben, Anm. 8), mag Rassismus u.a. auch in den Kriegsgefangenenlagern und vielleicht auch anlässlich jener dortigen anthropologischen Untersuchungen geortet haben. Einen Protest gegen jene, für die Betroffenen in vieler Hinsicht entwürdigenden biometrischen Praktiken formulierte jedenfalls am 31. Mai 1916 das IIC (dem Har Dayal allerdings zu dieser Zeit nicht mehr aktiv angehörte) in einem Schreiben an das Auswärtige Amt, unterstützt vom Indologen Heinrich Lüders: „The Sikhs especially will strongly resist on religious grounds any attempt made by the Europeans to touch any part of the body and more particularly the head. Further, such measurements are associated by Indians with criminals. We beg to warn the Government that the laudable scientific curiosity of German Professors will be attended with very unpleasant consequences." (AA Wk 11s. R 21256 Bd. 12 BI. 271, hier zitiert aus Lange 2013: 175f.). Doch dies muss Überlegung bleiben. Har Dayal, der die wissenschaftlichen Strömungen aufmerksam verfolgte, war vor dem Krieg Anhänger Ernst Haeckels, des berühmten, in Jena wirkenden Zoologen, Freidenkers und Pazifisten, der u.a. Eugenik und Rassenhygiene lehrte, und er empfahl in seinen Schriften jungen Indern, die Werke des Darwinisten zu lesen (vgl. Krüger 1964: 145). Nach Kriegsausbruch jedoch zeigte er sich in einem Brief enttäuscht über sein Idol (s.o., Anm. 3), vermutlich wegen Haeckels widersprüchlicher Einstellung zur deutschen Kriegspolitik. Gemeinsam mit anderen Größen der deutschen Kunst- und Wissenschaftsszene hatte jener kurz zuvor den problematischen Aufruf „An die Kulturwelt“ unterschrieben. Haeckel ließ sich in Folge zu weiteren nationalistischen und auch chauvinistischen bis rassistischen Äußerungen hinreißen, die mit seiner Vorkriegseinstellung unvereinbar schienen. 
Aufnahmen Har Dayals anwesend war (was jedoch bezeugt ist für jene, die im Phonogrammarchiv ein halbes Jahr später, im Herbst 1918, von Sirdar Umrao Singh Sher-Gil gemacht wurden, s.u.).

Sehr wahrscheinlich kannten die Wiener Wissenschaftler die politische Identität Har Dayals. Zum einen gibt er seinen wahren Namen im Archiv an (s.o., Abb. 1), zum anderen schreibt er in einem Brief vom 18. März an Bhupendranath Datta vom IIC in Berlin, dass er auf die Hilfe von Schroeders bei den Ausreiseplänen zählt: „I think I shall get the Danish visa with Prof. von Schroeder's help for ,scientific research' etc., and from there to Stockholm with Prof. Andersen's aid. Let us see how it turns out. Without direct official support, the matter becomes needlessly difficult." (Briefabschrift in AA Wk 11f. R 21112-2 Bl. 261). Von Schroeder war mit den deutschen Indologen, und gewiss mit Paul Deussen oder Heinrich Lüders, die mit dem IIC und dem Auswärtigen Amt in Berlin in Verbindung standen (s.o.), gut vernetzt.

Jedoch ist unklar, wieviel Kontakt Har Dayal tatsächlich mit Leopold von Schroeder hatte, der im Frühjahr 1918 mit schweren Herzproblemen zeitweise "krank darnieder lag", wie er in seinen Lebenserinnerungen (Schroeder 1921: 266) schrieb. Eine solche quasi politische Hilfe durch den Wiener Ordinarius wäre jedoch in der Atmosphäre von Vermischung von deutsch-nationalem, romantischem und indischem Gedankengut, die die damalige Indologie prägte und von Schroeder mitgetragen wurde, gut denkbar. Von Schroeder, der die Russifizierung seiner Heimatuniversität Dorpat/Tartu beklagt hatte, hoffte darauf, dass das Baltikum aus der russischen Krone ausgegliedert und sich Deutschland annähern würde, und dementsprechend freudig begrüßte er im Frühjahr 1918 die Besetzung seiner Heimat Estland durch die deutsche Armee (ibid.: 139ff. und 266). Jedoch brachten die Niederlage der Mittelmächte und die Unabhängigkeit Estlands noch im selben Jahr für von Schroeder große Enttäuschung bis zu seinem Tod 1920 (ibid.: Nachwort des Herausgebers, 267f.). ${ }^{16}$

Einerseits dem deutschnationalen Lager angehörig und den antisemitischen Lehren Houston Stewart Chamberlains aufgeschlossen, verkehrte andererseits der gläubige evangelische Christ von Schroeder freundschaftlich

Im selben Jahr 1918 erschien von Schroeders Lobschrift Houston Stewart Chamberlain - Ein Abriß seines Lebens, in dem der Autor u.a. auch seine persönliche Freundschaft zu dem englischstämmigen deutschnationalen Rassentheoretiker darlegt, dessen Werk später die nationalsozialistische Ideologie stark prägen sollte. Am 1. Oktober 1918 schloss von Schroeder seine Lebenserinnerungen $\mathrm{ab}$, die von seinen Erben unter seinen nachgelassenen Papieren gefunden und 1921 veröffentlich wurden (Schroeder 1921). Ebenfalls 1918 abgeschlossen hat von Schroeder das Manuskript „Die Gotteserkenntnis der Upanishaden und der Bibel", das aber nie publiziert wurde (vgl. ibid.: 264 und 281; Werba \& Griffiths 2006: 10, Anm. 22). 
mit Juden und anderen Nichtchristen (Schroeder 1921: 251). ${ }^{17}$ Sein Verhältnis zu den Studenten, auch zu den jüdischen wie Bernhard Geiger, muss sehr gut gewesen sein (ibid.: 225). 1907 legte sich von Schroeder in einem Schlagabtausch in der Presse auch mit dem mächtigen (und für seinen Antisemitismus bekannten) Wiener Bürgermeister Karl Lueger an (ibid.: 210). Als Dekan geriet Leopold von Schroeder an der Universität zwischen die Fronten der deutschnationalen und jüdischen Lager, als Feierlichkeiten zum 100. Geburtstag Richard Wagners in der Universität Wien im Mai 1913 anstanden, diese in Gewalt auszuarten drohten und der glühende Wagnerianer sich für die Seite der Burschenschaften engagierte (ibid.: 242f.). Im Phonogrammarchiv war von Schroeder eine gern gesehene Persönlichkeit, wie der damalige Assistent Hans Pollak viele Jahre später bezeugte. ${ }^{18}$

Har Dayals Urteil über von Schroeder ist nicht überliefert. Beide hätten viele gemeinsame Interessen gehabt. ${ }^{19}$ Ein Zeichen seiner Wertschätzung mag sein, dass Har Dayal wenige Monate vor seinem Tod in einer Aufzählung derjenigen namhaften Indologen, die er persönlich gekannt habe, gleich nach seinem Oxford-Lehrer Arthur Anthony Macdonell von Schroeder nennt, obwohl er den größeren Teil der danach genannten SanskritGelehrten schon Jahre vor von Schroeder kennengelernt haben musste. ${ }^{20}$

17 „[...] nur Arier und Semiten sind die Schöpfer großer Weltreligionen gewesen“, schrieb von Schroeder in der 1914 erschienenen Einleitung zu seinem dreibändigen Werk Arische Religion (S. 9); vgl. <http://dspace.utlib.ee/dspace/handle/10062/5329> (31.5.2014).

18 Der Germanist und Linguist Hans Pollak, der zwischen 1908 und 1927 Assistent im Phonogrammarchiv war, der gemeinsam mit Bernhard Geiger die Aufnahmen von Sirdar Umrao Singh im September 1918 erstellt hatte und 1938 wie Geiger aus Österreich vertrieben wurde, widmete von Schroeder in seinen nach dem Zweiten Weltkrieg in den 1970er-Jahren verfassten Erinnerungen zur Geschichte des Phonogrammarchivs einige freundliche Zeilen (Pollak 1973: 9): „[...] Professor Leopold von Schroeder [...], der damals wohl die denkwürdigste Gestalt der Wiener Universität war, eine wunderbare Verkörperung des Besten, was gewisse deutsche Adelige in den baltischen Provinzen dargestellt haben mögen. Er war ein Mann der guten alten Zeit, ein begeisterter Mythologe und Freund seiner Studenten. Schroeder interessierte stets der Inhalt, nicht die Sprachform als solche, und er las Sanskrit, als wäre es Deutsch."

19 Es sei hier an Har Dayals Aufsatz über die deutsche Kultur erinnert, in dem er großes Lob für Richard Wagner und Bayreuth ausdrückt (s.o., Anm. 11), und in dem es u.a. heißt, dass Bayreuth „in den kommenden Jahrhunderten eine Pilgerstätte für orientalische Musiker werden [wird] " (Dayal 1918: 264). Har Dayal geht allerdings nicht soweit, die Bühnenwerke Richard Wagners als Ausformung und Vollendung alt-indoiranischer Mythen und Mysterienspiele darzustellen, wie dies Leopold von Schroeder vor allem in seinem 1911 erschienenen Werk Die Vollendung des arischen Mysteriums in Bayreuth getan hat. Har Dayal mag die dem indischen Kulturkreis zugeschriebenen Elemente in Wagners Werk aufgefallen sein, so die Inhalte aus Parsifal, die dem Bodhisattva-Konzept entstammen sollen (vgl. dazu etwa Suneson 1989), zu dem er später seine Doktorarbeit schreiben sollte (Dayal 1932). 
Insgesamt ist Har Dayals Aussage, er könne mit Hilfe von Schroeders ein dänisches Visum bekommen, schwierig zu bewerten: Seine Briefe an seine Kollegen im Berliner Komitee wurden über das Auswärtige Amt geschickt und dort kontrolliert, womit Har Dayal rechnen musste (in der Tat ist der Brief vom Auswärtigen Amt geöffnet worden und sollte dann wieder verschlossen werden, ibid. AA Wk 11f. R 21112-2 Bl. 259f.). Womöglich hat Har Dayal mit diesem Satz Berlin zu entschlossenerem Handeln in der VisaFrage anstacheln wollen. Auch bleibt fraglich, ob das neutrale Dänemark bzw. der im Brief genannte Wissenschaftler Professor Andersen diese Hilfe geleistet hätte. Ein ähnlicher Plan wurde einige Monate später gefasst: Chattopadhyaya schrieb am 24. Juni 1918 aus Stockholm an von Wesendonk, er wolle den Indologen Prof. Johannson [Karl Ferdinand Johansson] bitten, Har Dayal als Assistenten in indischen Sprachen nach Uppsala anzufordern (bereits zuvor in einem Brief vom 27. Mai 1918 war dies erwogen worden, wobei die Frage im Raum stand, ob Johansson genügend deutschfreundlich sei). Doch dieser habe sich beschäftigt gezeigt und ihn auf eine Unterredung zu einem späteren Zeitpunkt vertröstet (AA Wk 11f. R 21115-2 BI. 4f. und R 21114-2 BI. 144).

Wenn auch Har Dayal offenbar seine Rolle als in Wien zu Studienzwecken weilender Akademiker erfüllt, so lässt ihn dies nicht von revolutionären Kreisen abhalten: Im zuvor erwähnten Brief vom 18. März an Bhupendranath Datta schreibt Har Dayal, er habe den Chef der ukrainischen Nationalisten getroffen, was inn auf die Idee gebracht habe, für die indische Sache eine sozialistische Zeitschrift zu gründen, den dafür nötigen sozialistischen Jargon beherrsche er, ",mit Zitaten von Marx etc. etc.", und er fügt hinzu, seiner Erfahrung nach seien nur die Sozialisten an Freiheit interessiert, allen anderen Parteien seien Asien und die Asiaten egal. Im Auswärtigen Amt vermerkte von Wesendonk handschriftlich dazu (AA WK 11f. R 21112-2 BI. 259ff.; vgl. auch Krüger 1964: 160f.):

Aus dem Brief, den Har Dayal aus Wien an das hiesige Indercomité ge[schickt?] hat und der hier ohne Wissen der Inder geprüft worden ist, geht wieder hervor, dass Har Dayal nur den Sozialdemokraten wahres Interesse

20 Vgl. den Brief an W. Norman Brown vom 24. November 1938: <http://www.saadigitalarchive.org/ item/20111011-404> (21.3.2014). Andererseits: Ist von Schroeder in den folgenden kritischen Worten Har Dayals (1920: 12f.) gemeint? „A learned professor, who is loved and respected by all his friends for his noble character, said in the spring of 1918: ,If this great offensive succeeds, there must be no more talk of evacuating Belgium. All that must be kept. It was part of the old German Empire." In dieser Passage zählt Har Dayal einige Beispiele von Aussagen auf, in denen sich die fehlgeleitete deutsche Junker-Arroganz, die er während des Weltkriegs kennengelernt habe, manifestiere. 
für Indien und Asien [überhaupt?] zutraut, übrigens ganz im Gegensatz zu dem Leiter des indischen Bureaus in Stockholm, Chattopadhyaya, der von den Sozialdemokraten nichts wissen will und nur an die Anarchisten glaubt.

Wiederum fragt sich, ob Har Dayal das Auswärtige Amt mit jenen Äußerungen provozieren wollte.

Auf der Suche nach einem Betätigungsfeld kommt im März das Gespräch auf jene Inder, die als Untertanen der britischen Krone auf österreichischungarischem Boden interniert sind. Zum Unterschied zu Deutschland waren in den Kriegsgefangenenlagern der k.u.k. Monarchie wegen der Frontverläufe keine Inder. Har Dayal sucht aber um Erlaubnis an, die indischen Zivilgefangenen, unter denen er angesehene Persönlichkeiten vermutet, besuchen zu dürfen, was durch das k.u.k. Ministeriums des Äußeren mit dem Hinweis gestattet wird, es seien nur zwei Inder in Österreich, beide seien Artisten und befänden sich im Zivilgefangenlager in Katzenau bei Linz (AA Wk 11f. R 21112-1 Bl. 36 und R 21114-1 BI. 29). Auch plant Har Dayal offenbar, laut einer handschriftlichen Note eines Diplomaten des Auswärtigen Amts, die Gründung eines Vereins zur Verbreitung der indischen Kultur sowie eine deutsche Übersetzung des Werks The Indian War of Independence über den indischen Aufstand von 1857, dessen Autor, der mit Har Dayal befreundete Revolutionär Vinayak Damodar Savarkar, zum Zeitpunkt Gefangener in der britischen Strafkolonie auf den Andamanen war (AA Wk 11f. R 21112-2 Bl. 276).

Im letzten Kriegsjahr herrscht überall Mangel. Offenbar erhoffen sich die indischen Kollegen vom IIC in Berlin, dass es in Wien noch besser sei, und bitten Har Dayal um Hilfe, denn im erwähnten Brief vom 18. März (AA Wk 11f. R 21112-2 Bl. 261) antwortet dieser: „I shall begin hunting for flannel for you. There is the Bezugscheinsystem here, but one can get things quietly in small shops."

Am 19. April schreibt das IIC an das Auswärtige Amt, Har Dayal wolle nicht nach Berlin zurückkommen, sondern in Südungarn oder Rumänien „zu einem längeren Erholungsurlaub“ bleiben (AA Wk 11f. R 21113 BI. 244). Ob es zu diesen Reisen kam, ist unklar, und es bleibt auch vorerst Vermutung, ob dieser Plan damit zusammenhing, dass seit Ende 1917 sukzessive die meisten indischen Kriegsgefangenen, die im Halbmondlager bei Wünsdorf interniert gewesen waren (s.o.), nach Südrumänien verlegt wurden (wo auch bis kurz vor Kriegsende die Studien des Indologen Heinrich Lüders, des Indogermanisten Wilhelm Schulze und des Anthropologen Rudolf Pöch fortgesetzt wurden, vgl. Lange 2013: 112-114). 
Sicher jedoch war Har Dayal zwischen Juli und Anfang August in Dunaharaszti bei Budapest zu Gast bei Sirdar Umrao Singh Sher-Gil und traf dort den inzwischen nach Europa zurückgekehrten Raja Mahendra Pratap. Pratap hatte 1915 zunächst seine Mission, nach Kabul zu gelangen, erfüllen können (s.o.) und dort die erste provisorische Exilregierung Indiens gegründet. Jedoch war ihm die Unterstützung des Emirs Habibullah bei den Aufwiegelungen in Indien gegen die britische Herrschaft versagt geblieben. Untätig hatte der Raja als Gast in Kabul verharren müssen, bis er schließlich über das bolschewistische Russland um den 22. März 1918 zurück nach Deutschland gelangt war (AA Wk 11f. R 21112 BI. 197, 199, 200, 204). Pratap ist enttäuscht von der Afghanistan-Mission und von der Zusammenarbeit mit Deutschland. Seine Begeisterung für den Bolschewismus hat in Berlin keinen Anklang gefunden (AA Wk 11f. R 21114-2 Bl. 152f.). Zunächst plant Pratap, sich aus der Politik gänzlich zurückziehen und sich in Budapest nur mehr seiner religiös-philosophischen und philanthropischen Bewegung einer Religion der Liebe zu widmen, wovon Umrao Singh Sher-Gil und Har Dayal inn abzuhalten versuchen. ${ }^{21}$ Tatsächlich ist Mahendra Pratap in seinem späteren Leben, vor und nach seiner Rückkehr nach Indien im Jahr 1946, politisch weiterhin engagiert gewesen.

Über Har Dayal schreibt Umrao Singh Sher-Gil im zuvor in Anm. 21 genannten Brief:

Mr. Hardayal was much starved in Wien, he was with me for several days. I was glad to see him and rub intellectual shoulders with him. When he is in more vigorous health after the more liberal dietary available in Hungary, I hope his wonderful mental powers will be utilized for the benefit of our respective countries India and Germany. He has been and is an extraordinary man, and I am glad to find that though in our religious philosophical ideas we stand far, we have come nearer. If one has love systems do not matter.

21 Wie aus einem Brief Umrao Singh Sher-Gils Anfang August an Glasenapp in Berlin (in Abschrift Glasenapps an das Berliner Auswärtige Amt vom 7. August, AA Wk 11f. adh R 21121-2 BI. 174-176) hervorgeht:

I had the pleasure and privilege of having the Kumar [= Mahendra Pratap] as my guest for a few weeks [...]. In opposition to our objections - mine, my wife's and Mr. Hardayal's, he is going to launch forth his religious career. We tried to show him what we honestly saw that few if any will listen or follow him. Often I and Hardayal made much friendly fun of some of his ideas, often we objected seriously, often with sorrowful sympathy we have admonished him. I and my wife have pathetically warned him, about the callousness of a selfish world [...]. He is firm, and says, 'If God wishes that it should succeed, it will, if not he [I] will learn by the result of experience that it was not God's will.' 
Inzwischen sind in Wien die Bemühungen, Har Dayal nach Stockholm zu bringen, intensiviert worden, denn in Schweden ist bereits im Mai der indisch-muslimische pro-britische Propagandist Yussuf Ali eingetroffen, und Har Dayal sei die richtige Person, diesem vor Ort entgegenzuwirken. Außerdem benötigt das Stockholmer Komitee Verstärkung, da Chattopadhyaya plant, nach Sankt Petersburg/Petrograd und Turkestan zu reisen. Am 24. September wird endlich die Einreisegenehmigung durch die schwedische Gesandtschaft in Wien ausgestellt (AA Wk 11f. R 21115-3 Bl. 254 und R 21115-4 Bl. 295), da ist Yussuf Ali allerdings bereits wieder abgereist. ${ }^{22}$ Anfang Oktober reist Har Dayal unter seinem wahren Namen, mit dem angegebenen Beruf "Universitätsprofessor", zum angeblichen Zwecke von literarischer Arbeit über die deutschen Grenzstationen Tetschen/Děčín und Sassnitz auf Rügen nach Schweden. ${ }^{23}$

Kurz zuvor sind Har Dayal und Umrao Singh Sher-Gil einander vermutlich ein weiteres Mal in Wien begegnet, denn am 21. September besuchte auch der Sirdar das Phonogrammarchiv und hinterließ drei Aufnahmen (Ph 1735-1737), in welchen er die Anfangsverse von Iśsopanișad, einige Verse der Bhagavadgītā und einige Lyrikstrophen auf Hindustani rezitierte (s.u., Kapitel 3). ${ }^{24}$ Von Schroeders Mitarbeiter Bernhard Geiger wird auf dem Aufnahmeprotokoll als anwesend vermerkt. ${ }^{25}$

Es gibt keine Hinweise darauf, dass Har Dayal das politische System der Donaumonarchie in besserem Licht als das wilhelminische Kaiserreich gesehen hat. Wenn er später von Österreich schrieb, dann nur in einem Atemzug mit Deutschland („the medieval dynasties of the Hohenzollerns and the

22 Zu Yussuf Ali in diesem Zusammenhang vgl. AA Wk 11f. R 21114-1 BI. 45, 50, 71f. etc.

23 Laut Personalausweis Nr. 5066 von Har Dayal (s.u., Abb. 2 und Abb. 3), ausgestellt am 8. August 1918 von der Kaiserlich Deutschen Paßstelle Wien I. Rotenturmstraße 19 (Politisches Archiv des Deutschen Auswärtigen Amts, Berlin, Bestand der deutschen Gesandtschaft in Stockholm, Aktenband „Indisches Nationalkomitee in Stockholm, Band 2", Nr. 133).

24 Umrao Singh Sher-Gil interessierte sich zeitlebens für technische Entwicklungen, insbesondere für die Fotografie, und beschäftigte sich auch nach dem Krieg mit Audiotechnik, wie ein Foto aus dem Jahr 1922 belegt, das nach der Rückkehr der Familie im nordindischen Simla aufgenommen wurde und auf dem er mit seiner jüngeren Tochter Indira mit Kopfhörer zwischen Edison-Phonographen und Schalltrichter zu sehen ist; s. <http://betterphotography.in/perspectives/great-masters/umraosingh-sher\%E2\%80\%91gil/14773/> (17.2.2015).

25 Möglicherweise war wiederum Professor von Schroeder an den Aufnahmen interessiert, der sich während seiner wissenschaftlichen Laufbahn immer wieder mit einer eigenen Übersetzung der Bhagavadgītā ins Deutsche beschäftigt und diese 1915 publiziert hatte. Beide, Umrao Singh SherGil und von Schroeder, waren literarisch tätig, verfassten und übersetzten Lyrik und trugen mit ihren literarischen und wissenschaftlichen Werken dazu bei, indische Literatur und indisches Gedankengut in Europa zu verbreiten. 
Hapsburgs", s.o., Anm. 7). Seine negative Sicht auf Deutschland war zweifellos bereits vor Wien entwickelt. Ob seine Erlebnisse in der k.u.k. Monarchie, seine Berühungen mit der Wiener Bürokratie und den in Österreich wirkenden politischen und gesellschaftlichen Kräften und Strömungen seine Einstellungen verschärft haben, lässt sich aus der Quellenlage nicht ableiten. Das Dreivierteljahr an der Donau verbrachte Har Dayal wohl in einem Zustand der „inneren Emigration“, und es nimmt sich biografisch - wie dies für seine Aufenthalte in Martinique und Waikiki gesagt wurde (Brown 1975: 84) - als Moratorium zwischen zwei Lebensphasen aus. ${ }^{26}$

Der Sirdar Umrao Singh Sher-Gil kehrte 1921 mit seiner Familie nach Indien zurück. Der Phonographist Har Dayals, der Physiker und spätere Direktor des Phonogrammarchivs Leo Hajek, wurde nach dem "Anschluss" Österreichs an Hitler-Deutschland 1938 aus Österreich vertrieben. Er erhielt einen Ruf an die Ohio State University und änderte seinen Namen zu Hayek. Vertrieben wurden auch der Assistent des Phonogrammarchivs Hans Pollak und der Indo-Iranist Bernhard Geiger. Geiger konnte seine Karriere noch 1938 in New York als Professor für Iranistik an der Columbia University und im Asia Institute, einer von Arthur Upham Pope gegründeten und geleiteten wissenschaftlichen Institution, fortsetzen. Pope, der vor dem Ersten Weltkrieg Dozent für Philosophie in Berkeley gewesen war, hatte seinerseits Har Dayal 1913 an der Universität von Kalifornien gekannt und gefördert. Theoretisch ist denkbar, dass Har Dayal, Geiger und Pope einander im Herbst 1938, als Har Dayal den Osten der USA kurz vor seinem Tod bereiste, in New York getroffen haben, sofern das alte Netzwerk zwanzig Jahre nach dem Ersten Weltkrieg noch bestand.

Währenddessen war in Europa jedenfalls ein neues Netzwerk im Entstehen: 1941 nahm Subhas Chandra Bose, der ebenso wie Har Dayal ein ehrenvolles Studium in Oxford für eine revolutionäre Laufbahn abgebrochen hatte, der auch wie jener militärische Mittel zur Erreichung der Freiheit befürwortete und damit in Distanz zu Gandhi geraten war, Zuflucht vor britischer Verfolgung in Deutschland. Bose, der in den Dreißigerjahren in Bad Gastein anlässlich eines Kuraufenthaltes eine Österreicherin kennengelernt und geheiratet hatte, sollte im Zweiten Weltkrieg eine neue Allianz mit dem deutschen Militarismus und der deutschsprachigen Indologie eingehen. Doch weder das eine noch das andere Bündnis mit Deutschland war es, das Indiens Unabhängigkeit erwirkt hat.

26 Es kann überlegt werden, dass der Kontakt zum Orientalischen Institut der Universität Wien und zum Phonogrammarchiv seinen Wunsch genährt hat, sich wieder der Wissenschaft zuzuwenden. 
3. Die Tonaufnahmen Har Dayals im Phonogrammarchiv ${ }^{27}$

Es gehörte im Phonogrammarchiv zum Aufnahmeprozess, die aufgenommenen Texte, ob gesprochen oder gesungen, soweit es möglich war, schriftlich zu fixieren. Die Protokollblätter zu Har Dayals Aufnahmen jedoch beinhalten nur Informationen über den Rezitator, über technische Details der Aufnahme, sowie das Datum und den Namen des Phonographisten. Texttranskriptionen fehlen, obwohl es für Har Dayal ein Leichtes gewesen wäre, seine Texte, die großenteils metrisch gebundene und bekannte Werke der Literatur darstellen, aufzuschreiben, nicht nur in den Ausgangsschriften (Devanagari bzw. arabische Schrift), sondern auch in lateinischer Umschrift. Vermutlich hatte es ein schriftliches Konzept der Aufnahmen oder zumindest eine Liste mit Titeln oder Beginnwörtern der geplanten Stücke gegeben, denn Har Dayal hat das „Programm“ der einzelnen Phonogramme mit ihren Texten offenbar gut geplant und die Stücke dann in einem Zug und zumeist auch ohne das unter den Aufnahmen dieser Zeit häufige Abbrechen mitten im Stück, wenn die Platte zuwenig Platz bot, rezitiert. Auch das Prosastück Vrṇdāvan Kathā (Ph 2901) ist flüssig ohne Verzögerung vorgetragen. Andererseits kann angesichts der hervorragenden Gedächtnisleistungen, die Har Dayal zeitlebens nachgesagt wurden (cf. Brown 1975: 30), nicht ausgeschlossen werden, dass er keine schriftliche Merkhilfe benützte.

In den Aufnahmeprotokollen von Sirdar Umrao Singh Sher-Gil (Ph 17351737) ist immerhin mit Bleistift vermerkt, aus welchen Werken rezitiert wurde (Íśāvāsya Upanișad und Bhagavadgītā). Das genauere Ausfüllen des Textfeldes scheint auf einen späteren Zeitpunkt nach dem Krieg verschoben worden zu sein. Doch weder für die eine noch die andere Aufnahmenserie gibt es irgendwelche Hinweise darauf, dass sie nach ihrer Entstehung jemals wieder beachtet worden wäre. ${ }^{28}$ Die Stimmporträts von Har Dayal und Sirdar Umrao Singh Sher-Gil gerieten für fast ein Jahrhundert in Vergessenheit.

27 Die Übersetzungen aus Hindi, Awadhi und Braj Bhasha (Ph 2900 und Ph 2901, Vrṇdāvan Kathā) wurden von Alaka Chudal gemacht, jene aus Urdu (Ph 2898, Ph 2902) von Stephan Popp; beiden sei auch Dank für viele sprachliche und historische Informationen ausgesprochen. Spezieller Dank gebührt zudem Suresh und Prerana Chandvankar für das Abhören und Identifizieren zahlreicher Texte. Der Textwortlaut aus Rāmcaritmānas 2 (Ayodhyākāṇ̣̂a), 51-52 von Tulsīdās (Ph 2901) wurde der Textsammlung GRETIL - Göttingen Register of Electronic Texts in Indian Languages, <http://gretil.sub. uni-goettingen.de/gretil/3_nia/hindi/tulrcm2u.htm> (5.6.2014), entnommen und angepasst; die englische Übersetzung wurde publiziert von Gita Press, <http://gitapress.org/BOOKS/1318/1318_ Sri\%20Ramchritmanas_Roman.pdf> (5.6.2014).

28 Im Unterschied zur Sammlung Felix Exners, zu der es schriftliche Publikationen und bessere Dokumentation gab und die bis ins 21 . Jahrhundert verschiedentlich in der wissenschaftlichen Literatur genannt wird. 
Die Phonogramme Har Dayals bieten Text- und Gesangproben aus dem literarischen Kanon der Sprache „Hindustani“ bzw. ihren linguistischen Varietäten Hindi, Urdu, Braj Bhasha und Awadhi. Die einzelnen Phonogramme lassen sich jeweils literarischen Gattungen bzw. Sprachen zuteilen: Auf Ph 2898 werden zwei patriotische Gedichte in Urdu gesprochen, darunter die berühmte Hymne Sāre jahāin se acchā (Tarāna-yi Hindī), verfasst von Muhammad Iqbal. Auf Ph 2899 singt Har Dayal ein zeitgenössisches Theaterlied in Hindi. Ph 2900 beinhaltet ein "Medley" gesungener Lyrik in Hindi, Braj Bhasha und Awadhi. Auf Ph 2901 ist Epik in Awadhi (metrisch, gesungen) und Hindi (gesprochene Prosa) zu hören. Ph 2902 stellt eine kleine Anthologie aus den Werken berühmter Urdu-Dichter dar. Der Literaturkenner und Philologe Har Dayal, der sich gerne öffentlich volksbildnerisch äußerte, wusste um die Ausdruckskraft der Poesie und hatte sie in seinen politischen und revolutionären Schriften bewusst eingesetzt. In der von Har Dayal gegründeten und geleiteten politischen Ghadr-Zeitschrift wurden Gedichte redigiert und gedruckt, die Zeitschrift hatte sogar einen Sammelband von Gedichten (Ghadr-kī-Ganj „Schatz der Revolution“) herausgegeben.

Im Folgenden sollen einzelne rezitierte Stücke herausgenommen und deren Texte (soweit bislang rekonstruiert) in Bezug zu Har Dayals Biografie und zum historischen Kontext gesetzt werden. Die Reihenfolge der hier beschriebenen Passagen entspricht nicht der Phonogramm-Nummer, die in jedem Fall unabhängig von der historischen Reihenfolge ist, in der die Aufnahmen erstellt wurden. Für eine umfassende Darstellung der rekonstruierten Texttranskriptionen sei auf die CD-Edition der Indischen Aufnahmen (1904-1929) im Rahmen der Complete Historical Collections 1899-1950 (in Vorbereitung) verwiesen. Die Auswahl der Texte stammt gewiss von Har Dayal selbst, denn es lässt sich - wie gezeigt werden wird - ein großer Teil der rezitierten Stücke und deren Inhalte mit Har Dayals persönlichen Einstellungen und Lebenslinien bis 1918 in Zusammenhang bringen. Dieselbe Wahlfreiheit zeigt sich auch bei den drei Aufnahmen von Sirdar Umrao Singh Sher-Gil (Ph 1735-1737), in denen der Beginn von İsopanișad (1-8), einige Verse der Bhagavadgītā (11.32-34, 47-50) sowie Hindustani-Lyrik, die auch aus der eigenen Feder stammen könnte, zu hören sind. ${ }^{29}$

29 Die Bhagavadgītā wurde im 19. Jahrhundert zu einem eminent wichtigen Text für den Neohinduismus und die indische Freiheitsbewegung. Aus ihr bezogen Gandhi und viele andere Rechtfertigung und Inspiration. Sie war auch für Umrao Singh Sher-Gil ein Leittext, wie aus einem Brief des Sirdars an von Wesendonk (vom 31. Oktober 1917) hervorgeht, in dem von einem weiteren Brief an Glasenapp die Rede ist, worin der Sirdar die gegenwärtige Kriegslage in Bezug zu den Lehren der Gītā erörtert habe (AA Wk 11f. R 21110-FN1 BI. 16ff., darin BI. 21). Zwar verfügte das Archiv bereits über Rezitationen der Gïtā unter den Exner-Aufnahmen, auf der anderen Seite bot Umrao Singh eine völlig andere Rezitationsweise. 
Im Unterschied zu Umrao Singh Sher-Gil rezitiert Har Dayal, obwohl selbst ein Sanskrit-Philologe, in Wien keine altindischen Texte in Sanskrit und Vedisch. Diesen zog er zumindest während seiner politischen Laufbahn das Studium neuerer Sprachen und Werke vor. „He never read the Vedas. He never bothered with the Gita", sagte sein Verwandter, der Publizist Gobind Behari Lal, später über inn (Brown 1975: 53). Den indischen Studenten riet Har Dayal, anstelle von Sanskrit lieber Hindi und moderne europäische Sprachen zu studieren (ibid.: 119) und sich mit modernen Wissenschaften anstelle von jahrtausendalten vedischen Texten und deren metaphysischen Inhalten zu beschäftigen (ibid.: 103). Den indischen Brahmanen, in deren Kompetenz die Überlieferung der altindischen Texte seit jeher gelegen hatte, wollte er verordnen, moderne Wissenschaften ins Sanskrit zu übersetzen (ibid.: 50).

Insofern passt es auch ins Bild, dass Har Dayal auf Ph 2901 nicht etwa das Sanskrit-Epos Rāmāyaṇa des Dichters Vālmīki, sondern aus dem Werk Rāmcaritmānas rezitiert, der späteren, von dem Dichter Tulsīdās vermutlich um 1574 u. Z. in Awadhi, einer frühen Varietät von Hindustani, verfassten Adaption des Rāma-Stoffes. Sanskrit war schon zu Tulsīdās' Zeit Literatursprache und den weniger gebildeten Schichten nicht geläufig, sodass der Dichter das Epos Rāmāyaṇa auch den weniger gebildeten Menschen zugänglich machen wollte. Insofern muss Tulsīdās für Har Dayal nicht nur für seine kunstvolle Sprache, sondern auch für seinen sozialen Ansatz zu bewundern gewesen sein, und in der Tat rangiert Tulsīdās in einer von Har Dayal 1938 publizierten Liste der wichtigsten Hindu-Persönlichkeiten (s.u.) an vierter Stelle. ${ }^{30}$ Har Dayal rezitiert auf Ph 2901 aus Rāmcaritmānas die Szene aus 2.51-52, in der Kronprinz Rāma, der durch Intrige und tragische Verstrickung für ein vierzehnjähriges Exil in den Wald geschickt wird, sich von seiner Mutter verabschiedet:

nava gayaṃdu raghubīra manu rāju alāna samān / chūța jāni bana gavanu suni ura anaṃdu adhikān // caupai: raghukulatilaka jori dou hāthā / mudita mātu pada nāya_u[?] māthā // dīnhi asīsa lāi ura līnhe / bhūșana basana nichāvari kīnhe // bāra bāra mukha cumbati mātā / nayana neha jalu pulakita gātā //

30 In seinem Artikel „The Indian Peasant“ (Dayal 1913), in dem er die Bildung und geistige Ausdruckskraft des indischen Proletariats, der Landarbeiter, Handwerker und Bediensteten, thematisierte, schrieb Har Dayal: „Who will give them a voice? Who will be their poet? Who will write a Ramayana and a Mahabharata for them? India awaits her true poet. For the people live in huts and hovels, not bungalows and palaces." (Brown 1975: 120). 
goda rākhi puni hrdayã[?] lagāe / stravata premarasa payada suhāe // premu pramodu na kachu kahi jāì / raṃka dhanada padabī janu pāì // sādara suṃdara badanu nihārī / bolī madhura bacana mahatārī // kahahu tāta jananī balihārī / kabahim lagana muda maṃgalakārī // sukrta sīla sukha sīvã suhāe [suhāī] / janama lābha ka_i avadhi aghāe [aghāī] //

The mind of Śrī Rāma (the Hero of Raghu's race) resembled a young elephant (newly caught) with kingship for its chain. When He heard of the proposal for exiling Him to the forest He took Himself as freed and felt overjoyed in His heart. (Caupai:) The Crown of Raghu's race, Śrī Rāma, joined both His palms and cheerfully bowed His head at His mother's feet. She blessed Him and clasped Him to her bosom and scattered jewels and raiment around $\mathrm{Him}$ (in order to protect Him from evil). The mother kissed His lips again and again with tears of affection in her eyes and her limbs thrilling over with joy. Seating Him in her lap she pressed Him once more to her heart, while milk flowed from her graceful breasts due to excess of love. Her affection and joy were altogether beyond description; it seemed as if a pauper had attained the position of Kubera (the god of riches). Fondly regarding His lovely countenance the mother spoke to Him in endearing terms; "Tell me, dear child - I beseech you; - when will be that delightful and auspicious hour, the beautiful culmination of piety, virtue and joy and the highest reward of human birth, - "

Auch das zweite Stück von Ph 2901 ist eine Wiedererzählung eines altindischen Textes, jedoch in zeitgenössischer Prosa. In dieser Hindi-Version von Vrṇdāvan Kathā, einer berühmten Episode des Bhāgavata Purāṇa, schickt der Held Krishna, nachdem er den feindlichen Kamsa (Kamsa, Kans) besiegt hat, seinen Freund Uddhav (Udho) in die Stadt Vrindavan, um seinen dort lebenden Zieheltern und den Gopis, den Hirtenmädchen und Gefährtinnen seiner Kindheit und Jugend, Nachricht von seinem Verbleib zu geben:

Śrī Śukdevjī bole to Prthvināth

jo Śrī Krșna Candrane Brndāvan kī sūrat karī.

To maim sab līlā kahtā hūṃ.

Tum citta de suno:

Ek din Hari ne Balrāmji se kahā ki bhāī sab Brṇdāvan basī hamārī suratkar atī dukh pāte hoṃge.

Jo ki jo ham ne unse avadhi dī thì wo bìt gayī.

Is se ab uchit hai ki kisīko vahã bhej dijīye

jo jākar un kā samādhān kar ā rahe. 
Yo bhāī se batākar [mațākar?], Hari ne

Udho ko bulāike kahā ki: Ho Udho

ek to tum hamāre bare sakhā ho

duje atī catur jñānvān aur dhīr.

Isliye ham tumhem Brndāvan bhejnā cahte hain

ki tum jākar Nand Yaśodhā aur gopiyon் ko jñān do.

Unkā samadhān kar āo

aur Mātā Rohīnī ko le āo.

Udho jī ne kahā jo āgayā.

Phir Śrī Krșna Candra bole ki tum pratham Nanda mahar

aur Yaśodhāji ko jñān upājae

unke man kā moh mițāe.

Aise samjhay kar kahilo.

Śrī Śukdevjī said: O Pṛthvināth, when Śrī Krsṣna Candra went to Vrindavan, I will tell you all his playful deeds (līlā). Listen with attention. One day Hari (Krșṇa) told Balrām: O brother, all inhabitants of Vrindavan might be worried thinking of us, because the time limit (of absence) we gave to them has been exceeded. Therefore better send somebody there who will solve their problem and returns. After speaking to his brother, Hari called Udho and said: O Udho, on the one hand you are our best friend, on the other you are very clever, wise and patient. Therefore we want to send you to Vrindavan, to go there and let Nanda, Yaśodhā and the gopīs (shepherdesses) know. You solve their problem (comfort them) and come back. And bring mother Rohīṇī (with you). Udho-jī said: As you say! Again Śrī Krșṇa Candra said: First you should go to chief Nanda and teach Yaśodhā to abandon her worldly attachments. This way you have to make her understand.

Die beiden Passagen, jene Abschiedsszene aus Rāmcaritmānas und die Erzählung Vrṇdāvan Kathā, in der sich der Held nach langen Kämpfen bei seiner Familie meldet, bilden wohl nicht zufällig gemeinsam den Inhalt der Aufnahme Ph 2901. Har Dayals drittes und letztes Buch Twelve Religions and Modern Life kam 1938, im Jahr vor seinem Tod, heraus. Darin zählt er die für ihn herausragendsten Gestalten und Persönlichkeiten des Hinduismus auf, darunter Swami Vivekananda, Aurobindo Ghose, seinen ehemaligen Lehrer Hans Raj, und den gemäßigten Politiker Gopal Krishna Gokhale, den Mentor Gandhis. Zuvor nennt er die beiden auf den Phonogrammen Ph 2900 und Ph 2901 vertretenen Literaten Kabīr (s.u.) und Tulsīdās. An der Spitze stehen die hinduistischen Heldengestalten Rama und Krishna (Brown 1975: 260f.).

Es wirkt, als ob der Exilant Har Dayal, der sein bürgerliches Leben und seine Familie um seiner revolutionären Aktivitäten willen verlassen und 
deswegen harte Kritik vor allem des Schwiegervaters auf sich gezogen hatte (vgl. Brown 1975: 48), sich sehr bewusst mit dem vorgetragenen Inhalt beider Texte identifizierte bzw. das eigene Leben und Tun in ein ähnliches Licht gerückt und damit gerechtfertigt sehen wollte. ${ }^{31}$ In Ramas Abschiedszene gewinnt beim Helden trotz des Schmerzes über die ungerechte Behandlung die Freude darüber, das harte Los anzunehmen, die Sohnespflicht zu erfüllen und dem Vater gehorsam zu sein, und über allem macht sich ein großes Gefühl der Befreiung breit, angesichts der Aussicht, ein Leben in der Wildnis abseits des Hofes zu führen. In Vrṇdāvan Kathā wiederum (Krishnas Beiname Hari ist einer der möglichen Ursprünge des ersten Namens Har Dayals, und die Stadt Vrindavan liegt nur etwa 145 Kilometer von Har Dayals Heimatstadt Delhi entfernt) ist in weiterer Folge der Geschichte die Freude der Familie über das Lebenszeichen des Helden schier grenzenlos. ${ }^{32}$

In beiden mythologischen Geschichten erklärt der Held seiner Mutter (Kauśalyā) bzw. Ziehmutter (Yaśodhā), dass sie ihren persönlichen Wunsch, den Sohn in der Nähe zu haben, für ein höheres Ziel (die Wahrhaftigkeit und pietas bzw. den Kampf gegen die Feinde) hintanhalten muss (s.o. und vgl. Rāmcaritmānas 2.51-56). Es erscheint kaum denkbar, dass Har Dayal bei der Rezitation nicht an sich und die eigene Familie in Indien denkt, auch angesichts der folgenden Zeilen, geschrieben im März 1919 in einem Amnestie-Gesuch an die britischen Behörden: „I wish to enquire how my mother, wife and daughter are. They will also be glad to have news of me and to learn that I have got rid of my old revolutionary ideas [...]" (Brown 1975: 221).

Krishna (Hari) und seine Gefährtin Rādhā, die Erste unter den Gopis, werden auch auf Ph 2900 (Nr. 6) mit einem ausdrucksvoll gesungenen Gedicht der indischen Mystikerin Mīrābāī genannt:

31 Während des Ersten Weltkriegs bestand zu seiner Frau Sundar Dayal und der gemeinsamen Tochter Shanti keine Nachrichtenverbindung. Hatte er seine Frau 1906 noch in sein studentisches Leben in Oxford einbeziehen können (gegen den Willen der Familie hatte Har Dayal sie nach England „entführt" und mit ihr bis Anfang 1908 dort gelebt; vgl. Brown 1975: 20f.), so wurde die Trennung durch seine fluchtartige Abreise aus Indien 1908 zu einer endgültigen.

32 Auf der Freude der Gopis über ihren Jugendgespielen ist das Konzept des Vishnuismus aufgebaut. Die positiven Gefühle, die der Bote mit seiner Nachricht hervorruft, werden im Vishnuismus als höchstes Verständnis der Gottesliebe angesehen. 
Srī rādhe rānī pe dāro na bāmsarī morī

Sonekī nahī rādhe rupekī nahī

Hare hare bāmskī korī

srī rādhe rānī pe daro na bāṃsarī morī

Mīrā ke prabhu girdhar nāgar

Bāmssarī lekar chorī

Do not play my flute to the holy lady Radha.

Radha is not made of gold, nor is she beautiful (or: of silver).

Oh lord Krishna, and she is only raw bamboo

Do not play my flute to the holy lady Radha

Mira's master is the holder of the mountain who finally/nevertheless takes the flute.

Har Dayal propagierte in seinen Schriften den Kulturaustausch zwischen Europa und Indien und riet jungen Menschen in Indien, europäische Sprachen wie Deutsch, Französisch und Englisch zu lernen, um selbst Einblicke in die Wissenschaft und Literatur Europas zu bekommen. Auf Ph 2899 verlässt er die ernste Literatur und singt das Theaterlied Pyār mohaniyā nibhānā hogā, ein Dialoglied von Mann und Frau aus dem Theaterstück Dil Farosh („Der Kaufmann/Verkäufer von Herzen“), einer Hindi-Adaption von Shakespeares Drama The Merchant of Venice. ${ }^{33}$

Auf Ph 2898 rezitiert Har Dayal zwei patriotische Gedichte, darunter das berühmte Ghazal Tarāna-yi Hindī von "Allāma" Muhammad Iqbāl (18771938), das nach dem Wortlaut der ersten Zeile auch unter dem Titel Sāre jahān se acchā (hindostān hamārā) „Besser als alle Welt ist unser Indien“ bekannt ist. Das Gedicht in Urdu wurde später vertont und wird bis heute ganz oder teilweise jährlich an jedem Unabhängigkeitstag gesungen. Muhammad Iqbāl hatte den Text 1904, als er am Government College in Lahore Philosophie unterrichtete, verfasst. Dort studierte auch der sieben Jahre jüngere Har Dayal (und kam erstmalig mit nationalen und politischen Bewegungen, wie dem Arya Samaj und dem Brahmo Samaj, in Kontakt; vgl. Brown 1975: 14ff.). Die erste Rezitation des Gedichts Tarāna-yi Hindī durch

33 Wie verbreitet das Lied vor dem Ersten Weltkrieg war, zeigt sich daran, dass der Liedtitel auch auf einer Liste der frühen Aufnahmen der Gramophone Company (1899-1908) erscheint; vgl. Kinnear (1994: 80; no. E1125, 14052). Demzufolge hatte es eine noch frühere Aufnahme des Liedes, gesungen von „Mr. Karua and Miss Mumtaz", gegeben. 
Iqbal selbst fand anlässlich einer von Har Dayal organisierten studentischen Zusammenkunft statt. ${ }^{34}$

Muhammad lqbal, der aus einer frommen und mystisch geprägten muslimischen Familie des Panjab kam, und Har Dayal, der der intellektuellen Hindu-Kaste Kayastha entstammte, waren von denselben Lehrern beeinflusste Philologen und hatten Philosophie studiert. In dem noch ungeteilten Hindustan waren die Sprachen Hindi und Urdu noch nicht durch unterschiedliche Nationalitäten mit-definiert. Bald nach der von Muhammad 'Umar Nūr-ilāhī geschilderten Rezitation von Sāre jahān se acchā reiste Iqual nach England, um in Cambridge zu studieren, und Har Dayal ging als BodenStipendiat nach Oxford. Doch während Iqbal sein Studium der Rechtswissenschaften und Philosophie abschloss bzw. ab 1907 in Deutschland fortführte und in München promovierte, legte Har Dayal sein von der britischen Kolonialregierung gewährtes Stipendium nieder und wurde antibritischer Revolutionär. Nach Deutschland gelangte Har Dayal erst 1915 infolge seiner politischen Zusammenarbeit mit dem Berliner Außenamt. Doch ob Student oder Agitator - von der deutschen Philosophie und Kultur waren beide, Muhammad Iqbal und Har Dayal, in hohem Maße beeindruckt.

Als Sāre jahān se acchā entstand, befand sich lqbal auf dem Höhepunkt seiner ersten Schaffensphase. Seine Gedichte aus dieser Zeit sind in Urdu geschrieben und zeugen noch von einer indisch-nationalistischen Grundhaltung. Die in diesem Zusammenhang vielfach zitierte Strophe 6 von Sāre jahān se acchā drückt eine patriotisch-säkulare Einstellung aus:

Mażhab nahīin sikhātā āpas men் bair rakhnā

Hindī hain ham vațan hai hindostāì hamārā

Religion lehrt nicht, einander feindselig zu sein.

Wir sind Inder, unsere Heimat ist Hindustan.

34 Gyan Chand fasst in Ibtida'i Kalam-i-lqbal (Chand 1988: 255f.) zusammen, was Muhammad 'Umar Nūr-ilāhī in seinem Artikel „Hindūstān hamārā kī shān-i nuzūl“ (etwa: „Die ehrenvolle Entstehung unseres Indien“), publiziert in der Zeitung $\bar{A} j \mathrm{kal}$ vom 1. Januar 1946, als ehemaliger Augenzeuge über die erste Rezitation von Tarāna-yi Hindī schreibt: Demnach war Har Dayal allseits beliebter Student und Mitglied im YMCA, hatte sich aber mit dem Vereinssekretär zerstritten und seinerseits die Young Men's Indian Association gegründet. Am Tag der Eröffnungssitzung ging er um drei Uhr nachmittags zu Iqbal, der damals Lektor an der Uni war, und überzeugte ihn, die Eröffnungsrede zu halten. Bei der Eröffnung um sechs Uhr trug Iqbal statt einer Rede sein Gedicht Sāre jahān் se acchā ohne Titel vor. Die Anwesenden waren begeistert und sprachen ihr Lob aus: „Da wir von diesem Gedicht Hilfe für unsere Mission bekommen haben, bedanken wir uns bei unserem alten Freund, Herrn Maulvi Muhammad Iqbal, und registrieren ihn im Verein." Muḥammad 'Umar Nūr-ilāhī war dabei, notierte sich das Gedicht und schrieb es nachher daheim ins Reine. Als das Gedicht später gedruckt wurde, kritisierte der Dichter Hasrat Mohānī das Urdu darin. Iqbal war sehr verärgert. 
Doch zurückgekehrt aus Europa, wo er sich die Texte von Hegel, Kant, Nietzsche, Bergson und Fichte erarbeitet hatte, widmete sich Iqbal zunehmend der islamischen Religion und ging daran, die Idee der Selbstverwirklichung, die von Swami Vivekananda für den Hinduismus entwickelt worden war, islamisch zu begründen. Iqbal wandte sich in seinen späteren Werken auf Urdu und Persisch vom Nationalismus ab und hin zum Panislamismus; die säkulare Einstellung von Sāre jahāin se acchā verschwand für immer aus seinem Werk. ${ }^{35}$

Iqbal beteiligte sich nicht an den indischen nationalistischen Aktivitäten während des Ersten Weltkriegs, engagierte sich jedoch in der Muslim League und unterstützte in den 1920er-Jahren als der muslimische Intellektuelle par excellence entschieden den späteren Gründer von Pakistan, Muhammad Ali Jinnah. Iqbal war der Erste, der öffentlich in einer Rede als Präsident der Muslim League von einem separaten muslimischen Staat in Nordwestindien sprach, und wurde damit zum Vordenker und nationalen Literaten Pakistans. Sein Frühwerk Sāre jahāni se acchā, das Teil der Landeskultur Indiens wurde, hat Iqbal nie verleugnet. 1924 wurde es in seine Gedichtsammlung Bānig-i Darā aufgenommen.

Während Sirdar Umrao Singh Sher-Gil, der ebenfalls vor 1908 in Lahore gelebt und Iqbal gekannt hatte, dem Dichter freundschaftlich verbunden blieb (1933 machte er berühmte Porträtfotografien von lqbal), ist von weiterem Kontakt zwischen Iqbal und Har Dayal nichts bekannt; zu Har Dayals komplexer Einstellung zum Islam s.o. Was Ph 2898 betrifft, so erscheinen auf dieser Aufnahme mit der Rezitation von lqbals Sāre jahān se acchā durch Har Dayal und angesichts der Kreuzungen und Gegenläufe in den Lebenswegen von Dichter und Rezitator - Moslem und Hindu, beide politische und literarische Menschen - in übertragenem und weitem Sinn bedeutende Teile der Anti-Kolonialbewegung und -geschichte Indiens vereint.

Der von Har Dayal gesprochene Text unterscheidet sich von der heute üblichen Version. Har Dayal zieht die Strophe 5 den Strophen 3 und 4 vor. Die Strophe 8 ist ausgelassen, wie das auch bei heutigen Rezitationen häufig gemacht wird. Nach sprachlicher Kritik hatte Iqbal die ursprüngliche Version dieser vorletzten Strophe überarbeitet. Doch hatte Har Dayal im Jahr 1918 vermutlich keine Kenntnis von der verbesserten Version.

35 1910, sechs Jahre nach Sāre jahān se acchā oder Tarāna-yi Hindī, dichtete Iqbal das Ghazal Tarāna-yi millī mit demselben Reimschema und derselben Silbenzahl pro Vers wie Tarāna-yi Hindī sowie weiteren inhaltlichen und sprachlichen Ähnlichkeiten, in dem er nicht die Einwohner Indiens, sondern die Moslems in der ganzen Welt ansprach. 
Auch die letzte (ursprünglich neunte) Strophe des Gedichts hat Iqbal geändert; sie lautet heute:

Iqbāl! koī mahram apnā nahīn jahān men்

Mālūm kyā kisī ko dard-i nihān hamārā

Iqbal, wir haben keinen Vertrauten in der Welt.

Kennt irgendjemand unseren verborgenen Schmerz?

Har Dayal kennt auch diese Korrektur nicht und rezitiert offenbar aus dem Gedächtnis die Version, die lqbal an dem Studentenabend vortrug, also im Wesentlichen (wenn auch mit der geringfügigen Wortumstellung apnā maḥram koī nahìn "to us, there is no confidant" anstatt koi mahram apnā nahïn "there is no confidant to us") die originale Version Iqbals: ${ }^{36}$

lquāal! apnā mahram koī nahīn jahāñ men்

Mālūm hai hamī ko dard-i nihān hamārā

Iqbal! Unser Vertrauter ist niemand in der Welt.

Kennen wir unseren verborgenen Schmerz?

An der in der Originalfassung stehenden Zeile mālūm hai hamen் (hamī) ko dard-i nihān hamārā („Do we know our hidden pain?" oder "We know our hidden pain") wurde beanstandet, dass der Possessivanzeiger hamārā, der in diesem Gedicht als radīf (= der für sämtliche Zeilen festgesetzte und verbindliche Reimschluss) verwendet ist, grammatisch schlecht verträglich sei mit dem hier als Reflexivum gebrauchten hamen ko (hier hamī ko). Die Partikel ko ist hier redundant und dürfte nur infolge der korrekten Silbenzahl verwendet sein. In klassischem Urdu reicht als Akkusativobjekt hamen.

Mit einem patriotischen Lied beginnen auch die Lyrikproben auf Ph 2900. Die Zeilen erinnern stark an die erste Strophe der französische Marseillaise und dürften mehr oder weniger eine Übertragung darstellen. Har Dayal war selbstverständlich von der Französischen Revolution beeindruckt und diskutierte 1908 in Kanpur darüber viel mit seinen Verwandten Gobind Behari Lal und Tara Chand (Brown 1975: 49).

36 Zitiert nach <http://www.chapatimystery.com/archives/univercity/examining_iqbal.html> (15.5.2014). 
Calo tum deś ke sab jan, fatah kā ā gayā ab din

Jhanḍ̂̄a[?] zulm kā khūnī kharāa hai rubarū apnī

Use ho maidan mem ān, duśman maine ki lalkār

Auf, geht, alle Leute der Heimat, jetzt ist der Tag des Siegs gekommen. Die blutige Fahne der Tyrannei steht uns gegenüber.

Man muss am Schlachtfeld stolz sein, wenn/dass man den Feind herausgefordert hat.

Ph 2902 bietet eine kleine Anthologie berühmter Urdu-Lyrik der Dichter Znauq, Inshā' und Mīr Taqī Mìr. ${ }^{37}$ Har Dayal hatte selbst für die Zeitschrift Ghadr in Urdu geschrieben, dabei die englischen Übersetzungen seiner Texte bemängelt und selbstbewusst bemerkt, besser sollte er selbst die Übersetzungen machen (Brown 1975: 145-147). Mit der Auswahl der genannten Dichter und Werke stellt der Rezitator auf Ph 2902 auch seine Geburtsstadt Delhi und den Panjab, seine Hauptwirkungsstätte innerhalb Indiens, dar. Im zweiten Gedicht, einem Zweizeiler, der infolge der Namensnennung des Dichters wohl den Schluss eines Ghazals bildet, drückt Zauq einerseits seine Wehmut nach Delhi aus, andererseits stellt er sich stolz und ironisch - vermittels eines Wortspiels zu seinem Namen - als einzigen dar, der Geschmack habe. Die Worte könnten auch Har Dayals Schicksal und Persönlichkeit abbilden:

Hai shakl meñ ājkal dard-i muhar faqre sakūn Kauñ jāe Żauq kar Delhi ki galiyāin choŕkar

Es ist gerade so, dass ich heutzutage den Schmerz des Siegelrings (= Kuss) zu einem Satz machen kann: Wer ist „Zauq“-voll (wörtlich: „Geschmack-voll“) gegangen/geworden und hat die Gassen Delhis verlassen?

Der folgende Zweizeiler ist quasi ein selbstgeschriebener Nekrolog des Dichters Zauq:

Kahte hain āj Zֵauq jahān se gužar gayā

Kyā khūb ādmī thā, Khudā mag்firat kare

37 Die Aufnahme ist leider noch nicht in allen Einzelheiten textlich geklärt. 
Man sagt, heute ist Zauq aus der Welt gegangen. ${ }^{38}$

Was für ein guter Mensch war der, Gott verzeih ihm.

Unmittelbar darauf wird ebenfalls in einem Gedicht von Zauq der Panjab genannt, jene Landschaft des damaligen Indien, mit dem Har Dayals Biografie nach Delhi am engsten verbunden ist: Zum einen hatte Har Dayal dort studiert und Kontakte zu nationalistischen Kreisen geschlossen, zum anderen war es das Land, das er durch seine revolutionäre und publizistische Tätigkeit im Jahr 1908 am meisten geprägt hatte. Die von ihm mitgeplante Revolte im ersten Halbjahr von 1915 war im Panjab ausgebrochen und niedergeschlagen worden.

Panjāb meñ bhī ab na rahī āb-u tāb-i husn
Ai Žauq, pānī ab to vo Multān bah gayā
Ye roē phūt'phūt'ke pāon ke āblae [ublae?]
Nāla sā ek soy-i beābān bah gayā

Im Panjab ist der Schein (oder: Wasser, trad. Wortspiel) und Glanz der Schönheit auch nicht mehr da. Oh Zauq, das Wasser (als Ausdruck für Tränen?) ist jetzt nach Multan geflossen. Er weinte bitterlich und benetzte die Füße. Wie ein Kanal/Bach ist es zur Wüste geflossen.

Vom Dichter Sayyid Inshā’ Allāh Khān, genannt „Inshā'“ (1756-1817), rezitiert Har Dayal zwei Gedichte. Wie Har Dayal war Inshā' polyglott und konnte mühelos zwischen den Varietäten von Hindustani wechseln. Inshā' ist auch als Verfasser der ersten Grammatik des Urdu bekannt. Nach dem die Waffenbrüderschaft thematisierenden Gedicht Kamar bāndhe hūe chalne ko yān sab yār baithe hain spricht Har Dayal einen Zweizeiler mit klassischem Selbstlob des Dichters:

Inshā' kī ġazal jisne sunī usne kahā vāh

Kyā bāt hai kya bāt hai kya bāt hai vallāh

Wer Inshas Ghazal hört, der sagt „,hui!“

Was ist los, was ist los, was ist los (wie gut ist das), oh Gott!

38 Oder (wegen Doppeldeutigkeit von jahān = persisch „Welt" oder Urdu-Relativpronomen „wo"): „Man sagt, wo Zauq heute vorbeigegangen ist". 
Stolz auf das eigene dichterische Können spricht auch aus dem folgenden Stück von Mìr Taqī Mīr, einem weiteren Urdu-Dichter aus Delhi, der im 18. Jahrhundert wirkte. Es handelt sich wohl um den Schluss eines traurigen Ghazals:

Āre Mīr ke āhistā bolo abhī kūch rote rote so gayā hai

Oh Mir, sprich behutsam/liebenswürdig, jetzt ist jemand weinend eingeschlafen.

Der Publizist Gobind Behari Lal, ein Verwandter und zeitweiliger Weggefährte Har Dayals, schilderte, wie Har Dayal während seiner Zeit in Berkeley ebenfalls aus dem Werk Mīr Taqī Mīrs auswendig rezitiert hatte: Am 25. Dezember 1912 erreichte die indischen Studenten die Nachricht jenes Attentats in Delhi, bei dem der Viceroy Lord Hardinge verletzt worden war; vgl. Brown (1975: 131):

When news of the bombing attempt was circulated, the students shouted, danced, and sang "Bande Mataram". Har Dayal, at his most eloquent, was the principal speaker. He concluded his stirring remarks by quoting a well-known line from the work of the great Urdu poet, Mir: "Pagri apni sambhaliyeago ...! Aur basti nahin, yeh Dilli hai!" Roughly translated, this means "Watch your step! This is not just any town, this is Delhi!'39

Ein weiteres Beispiel solch literarischer Schlagfertigkeit findet sich vermutlich auf Ph 2900 (Nr. 2), mit einem doha (Couplet) des religiösen Dichters („Sant“) Kabīr (1440-1518) in Awadhi.

Raghukul rīt yahī calī āì prān jāe par vacan

Raghukul rìt yahī calī āì prān jāe par vacan na jāì

Hier kommt die Sitte von Raghukul (= Rāma) zur Geltung - der Atem vergeht (bzw. man stirbt), aber das Wort geht nicht weg (bleibt bestehen).

Dieses in Indien auch heute sehr bekannte und im täglichen Leben oft zitierte Gedicht drückt den Willen aus, bis zum Tod und darüber hinaus zu

39 Das Attentat wurde von den britischen Behörden mit Har Dayal in Zusammenhang gebracht, und auch Har Dayal selbst deutete in seinem Bekanntenkreis eine Verbindung zu dem Attentäter an. Von da an drohte Har Dayal, wäre er nach Indien oder an einen anderen Ort unter britischer Judikatur eingereist, eine lange Haftstrafe, wenn nicht gar die Todesstrafe. 
seinem Wort zu stehen. Der Held Rama und das Epos Ramayana bildeten für Har Dayal und seine Anhänger geistige Eckpfeiler der nationalistischen Bewegung. Als der Oxford-Student den Sommer 1906 in Delhi verbrachte, wurde er gebeten, in seiner früheren Schule, dem St. Stephen's College, zu unterrichten. Auch dort gründete er eine studentische Vereinigung, den „Ramayana-Club“ (Brown 1975: 20). Später verglichen Har Dayal und seine Anhänger Rama mit „Self-Rule“, also dem Konzept einer nationalen und republikanischen Regierung für Indien. Den Dämon Ravana setzten sie mit dem britischen Imperialismus gleich, Ramas getreue Frau Sita mit Indien (Brown 1975: 10f.). Gandhi wiederum forderte die Etablierung von Ram raj (also der Herrschaft Ramas) im Sinne einer moralischen Kraft (Brown 1975: 76). Doch darüber hinaus hat Har Dayal diesen zum Zeitpunkt der Aufnahme mindestens 400 Jahre alten Zweizeiler Kabīrs vermutlich bewusst und humorvoll gewählt, um damit anachronistisch die ihn zum Zeitpunkt der Aufnahme umgebende, noch junge Audiotechnik zu umschreiben.

Auch wenn die von Har Dayal rezitierten Texte derzeit noch nicht in allen Einzelheiten identifiziert sind, zeigt sich klar, dass sie von ihm für die Tonaufnahmen eigenständig ausgewählt wurden. Selbstverständlich lässt sich nicht mit Sicherheit sagen, ob Har Dayal bei der Rezitation von Rāmcaritmānas und Vrṇdāvan Kathā auf Ph 2901 bewusst an seine Familie in Indien gedacht hat, noch, ob er bei dem wehmütigen Gedicht Panjāb meñ bhī ab na rahī $\bar{a} b-u$ tāb-i husn auf Ph 2902 den von inm zwei Jahre zuvor geplanten und fehlgeschlagenen Aufstand im Panjab im Sinn hatte. Jedoch scheinen in der Wahl der Stücke Bezüge zur eigenen Biografie deutlich durch, wie etwa mit der Hymne Sāre jahān se acchā, bei deren erster Rezitation durch den Dichter Iqbal Har Dayal anwesend war. Die Audiotechnik, die an jenem 7. Februar 1918 in Wien äußerlich dazu diente, der österreichischen Wissenschaft Sprachproben von Hindustani zu vermitteln, wird von Har Dayal gleichzeitig als privates Ausdruckmittel genutzt. Neben den vielen politischen, soziologischen, historischen, aufwiegelnden, theoretischen, revolutionären, propagandistischen, pädagogischen und philanthropischen Schriften, die der indische Unabhängigkeitsaktivist zeit seines Lebens verfasst hat, bieten die Tonaufnahmen im Wiener Phonogrammarchiv ein sehr persönliches Vermächtnis Har Dayals. Nicht nur durch die festgehaltene Stimme und die akustische Interpretation, sondern auch durch die in den rezitierten Texten dargestellten Gefühle und Inhalte wirken die Aufnahmen persönlicher als viele von Har Dayals eigenen Schriften, obwohl die rezitierten Texte zur Gänze oder zumindest zum allergrößten Teil nicht von Har Dayal stammen. 
Auf der anderen Seite scheint Har Dayal das junge Medium zu nützen, um sich und seine revolutionären Aktivitäten, die nicht nur innerfamiliär, sondern auch durch andere Vertreter der indischen Freiheitsbewegung kritisiert worden waren, und die er in der gegenwärtigen Lebensphase infolge der bereuten Verbündung mit Deutschland wohl zum Teil selbst nicht mehr ungetrübt sah, durch die Assoziierung mit den indischen Helden Rama und Krishna zu erklären und möglicherweise zu rechtfertigen. Anfang Februar 1918 rechnete Har Dayal damit, sehr bald dem Einflussbereich der Mittelmächte, die er zu verachten und misstrauen gelernt hatte, zu entkommen. Die andere politische Welthälfte, das Britische Empire mit seinen Verbündeten, war ihm schon lange versperrt.

Har Dayal konnte sich demnach über sein weiteres Schicksal nicht klar sein. Insofern kann der Revolutionär die Auswahl mehr oder weniger bewusst als ein von ihm sorgsam zusammengestelltes Vermächtnis angesehen haben, das unveränderbar im Phonogrammarchiv in Form einer Metallmatrize gelagert werden würde. Es ist die Auswahl des gebildeten literarischen Menschen Har Dayal, mit der er sich selbst - nicht nur der Wissenschaft, sondern wohl auch der übrigen Welt und Nachwelt - darstellt. Selbst die Aufnahmesituation am 7. Februar 1918 ist von ihm literarisch umschrieben durch das Gedicht Kabirs in Alt-Hindustani:

Raghukul rīt yahī calī āī prān jāe par vacan na jāī

Hier kommt die Sitte von Rama zur Geltung - der Atem vergeht, aber das Wort bleibt bestehen. 


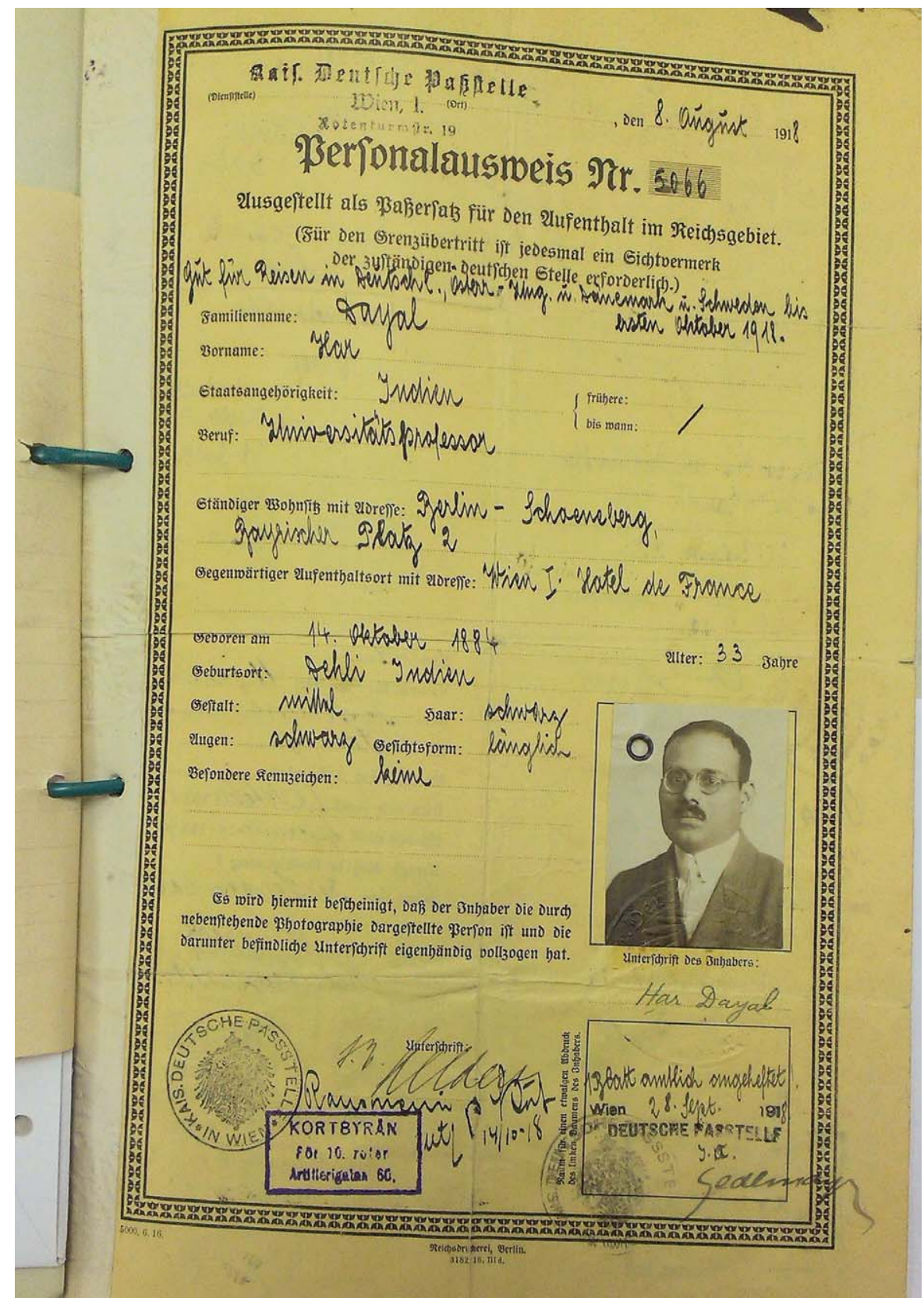

Abb. 2: Personalausweis von Har Dayal, Vorderseite (Abb. 2 u. 3 mit freundl. Genehmigung des Politischen Archivs des Deutsches Auswärtigen Amts, Berlin) 


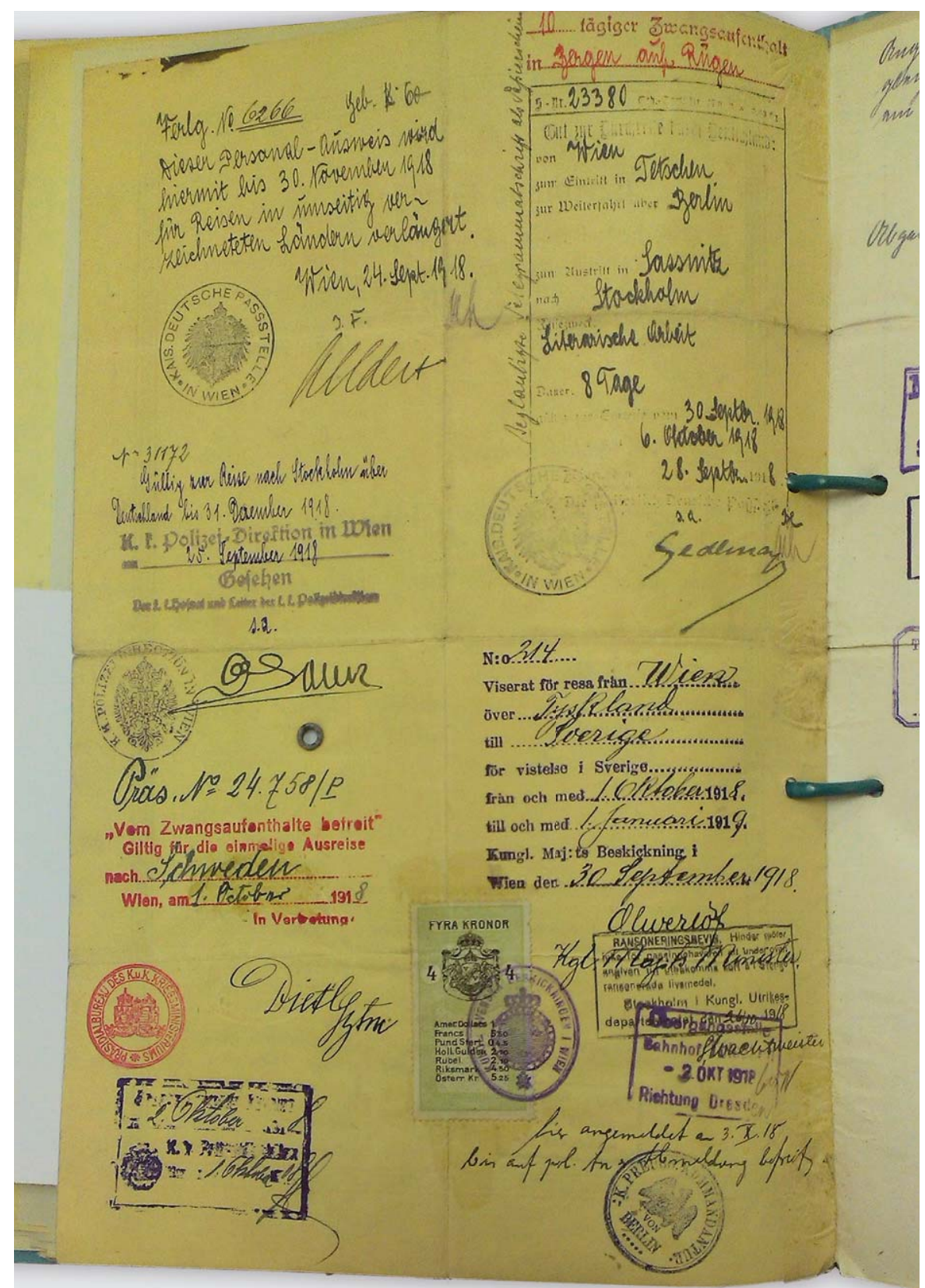

Abb. 3: Personalausweis von Har Dayal, Rückseite 


\section{Literaturverzeichnis}

Bihl, Wolfdieter. 2009. Orientalistik an der Universität Wien. Forschungen zwischen Maghreb und Ostund Südasien: Die Professoren und Dozenten. Wien etc.: Böhlau.

Brown, Emily C. 1975. Har Dayal: Hindu Revolutionary and Rationalist. Tucson: The University of Arizona Press.

Chand, Gyan. 1988. Ibtida'i kalam-i lqbal. Bah tartib mah o sal. Haidarabad \& Dihli: Urdu Research Centre.

Dayal, Har. 1912. „Karl Marx: A Modern Rishi“. The Modern Review XI (3): 43-50.

Dayal, Har. 1913. „The Indian Peasant“. The Modern Review XIII (5): 506-509.

Dayal, Har. 1918. „Der Orient und die deutsche Kultur“. Der neue Orient 3 (6; 19. Juni 1918): 261-265.

Dayal, Har. 1920. Forty-Four Months in Germany and Turkey: February 1915 to October 1918. A Record of Personal Impressions. London: King \& Son.

Dayal, Har. 1932. The Bodhisattva Doctrine in Buddhist Sanskrit Literature. London: Kegan Paul, Trench, Trübner \& Co.

Dayal, Har. 1934. Hints for Self-Culture. London: Watts \& Co

Exner, Sigmund. 1918. „Gemeinsame Phonogrammarchiv-Kommission: Tätigkeitsbericht über das Jahr 1917/18“. Almanach für das Jahr 1918/Akademie der Wissenschaften in Wien (68. Jg.): 301-303.

Felber, Erwin (mit Texten und Übersetzungen von Bernhard Geiger). 1912. Die indische Musik der vedischen und der klassischen Zeit: Studie zur Geschichte der Rezitation. Nach den Platten des PhonogrammArchives der kais. Akademie. (Sitzungsberichte der Kais. Akademie der Wissenschaften in Wien, Philosophisch-Historische Klasse, 170/7 = XXIII. Mitteilung der Phonogramm-Archivs-Kommission). Wien: Hölder.

Kinnear, Michael. 1994. The Gramophone Company's First Indian Recordings (1899-1908). Bombay: Popular Prakashan.

Krüger, Horst. 1964. „Har Dayal in Deutschland“. Mitteilungen des Instituts für Orientforschung X (1): 141-169.

Lange, Britta. 2013. Die Wiener Forschungen an Kriegsgefangenen 1915-1918: Anthropologische und ethnografische Verfahren im Lager. (Österreichische Akademie der Wissenschaften, Philosophisch-Historische Klasse, Sitzungsberichte, 838). Wien: VÖAW.

McGetchin, Douglas T. 2010. „Indo-German Connections, Critical and Hermeneutical, in the First World War". The Comparatist 34: 95-126.

Paul, E. Jaiwant \& Shubh Paul. 2003. Har Dayal - The Great Revolutionary. New Delhi: Roli Books.

Pollak, Hans. 1973. „Von Dr. Hans Pollak geliefertes Material zur Benutzung nach Wunsch für die Verfasser der Geschichte des Wiener Phonogrammarchivs" (Typoskript, 10 S.).

Schroeder, Felix von (Hg.). 1921. Lebenserinnerungen von Leopold von Schroeder Dr. phil. et theol. h.c. weil. Professor an der Universität Wien und Mitglied der Wiener Akademie der Wissenschaften. Leipzig: Haessel.

Suneson, Carl. 1989. Richard Wagner und die indische Geisteswelt. Leiden: Brill.

Werba, Chlodwig H. \& Arlo Griffiths. 2006. „Leopold von Schroeder: Eine annotierte Bibliographie“. Wiener Zeitschrift für die Kunde Südasiens/Vienna Journal of South Asian Studies 50: 5-25.

\section{Ulla Remmer: Sprachwissenschaftlerin, Phonogrammarchiv der ÖAW.}

